\title{
Targeting IL-17A/glucocorticoid synergy to CSF3 expression in neutrophilic airway diseases
}

\author{
Suidong Ouyang, Caini Liu, Jianxin Xiao, Xing Chen, Andy C. Lui, and Xiaoxia Li \\ Inflammation and Immunity, Cleveland Clinic, Cleveland, Ohio, USA.
}

IL-17A plays a critical role in the pathogenesis of steroid-resistant neutrophilic airway inflammation, which is a hallmark of severe asthma and chronic obstructive pulmonary disease (COPD). Through RNA sequencing analysis of transcriptomes of human airway smooth muscle cells treated with IL17A, dexamethasone (DEX, a synthetic glucocorticoid drug), alone or in combination, we identified a group of genes that are synergistically induced by IL-17A and DEX, including the neutrophilpromoting cytokine CSF3. In type-17 (Th17/IL-17A ${ }^{\text {hi }}$ ) preclinical models of neutrophilic severe asthma (acute and chronic) and COPD, although DEX treatment was able to reduce the expression of neutrophil-mobilizing CXCL1 and CXCL2 in lung tissue, CSF3 expression was upregulated by DEX treatment. We found that DEX treatment alone failed to alleviate neutrophilic airway inflammation and pathology, and even exacerbated the disease phenotype when CSF3 was highly induced. Disruption of the IL-17A/DEX synergy by IL-17A inhibition with anti-IL-17A mAb or cyanidin-3glucoside (C3G, a small-molecule IL-17A blocker) or depletion of CSF3 effectively rendered DEX sensitivity in type-17 preclinical models of neutrophilic airway diseases. Our study elucidates what we believe is a novel mechanism of steroid resistance in type-17 neutrophilic airway inflammation and offers an effective steroid-sparing therapeutic strategy (combined low-dose DEX and C3G) for treating neutrophilic airway diseases.

Authorship note: SO and CL contributed equally to this work.

Conflict of interest: The authors have declared that no conflict of interest exists.

Copyright: (c) 2020, American Society for Clinical Investigation.

Submitted: August 27, 2019

Accepted: January 8, 2020

Published: February 13, 2020.

Reference information: JCI Insight. 2020;5(3):e132836.

https://doi.org/10.1172/jci.

insight.132836.

\section{Introduction}

IL-17A, a major proinflammatory cytokine produced by Th17 cells, has been implicated as an important cytokine contributing to the pathogenesis of steroid-resistant neutrophilic airway diseases including severe asthma and chronic obstructive pulmonary disease (COPD) (1-5). IL-17A induces an array of cytokines/ chemokines (e.g., CSF2, CXCL1, CXCL2, CSF3, IL-6, and IL-8) to elicit neutrophilic airway inflammation. Although IL-17A neutralization showed no effect on type-2 (Th2) responses in asthma patients or preclinical models, blocking IL-17A showed beneficial effects on type-17 (Th17/IL-17A ${ }^{\text {hi }}$ ) steroid-resistant neutrophilic asthma and COPD (3, 6-10). However, the molecular mechanisms for the observed association between steroid resistance and Th17/IL-17A-driven airway neutrophilic inflammation remain poorly understood.

Glucocorticoids (GCs) are commonly used antiinflammatory therapeutic reagents to treat asthma and COPD. GCs bind the glucocorticoid receptor (GR, encoded by NR3C1) and the activated GC/GR complex may repress inflammatory gene transcription by repressing binding (transrepression) of other transcription factors (e.g., AP1 and NF- $\mathrm{kB}$ ) to inflammatory genes or by directly activating transcription (transactivation) of multiple antiinflammatory genes $(11,12)$. Although GC-based drugs can effectively manage Th2 inflammation via inducing apoptosis of Th2 cells and eosinophils and inhibiting type-2 cytokines, they have little efficacy in inhibiting Th17/IL-17A ${ }^{\text {hi }}$ neutrophilic severe asthma and COPD that are steroid resistant or refractory $(7,13-15)$.

In the current study, we demonstrated that IL-17A and dexamethasone (DEX, a synthetic GC drug) synergistically induced the expression of a group of genes including colony-stimulating factor 3 (encoded by CSF3 and also known as granulocyte colony-stimulating factor, G-CSF) in airway smooth muscle cells (ASMCs) via both transcriptional and posttranscriptional mechanisms. CSF3 is an important neutrophil-promoting cytokine that affects many aspects of neutrophils including proliferation and survival. By employing Th17/IL-17A $\mathrm{A}^{\text {hi }}$ preclinical mouse models of neutrophilic severe asthma (acute and chronic) and COPD, we showed that Csf3 expression was substantially induced in the lung tissue by DEX treatment, although the expression of neutrophil-mobilizing $C x c l 1$ and $C x c l 2$ was decreased. 
Furthermore, DEX treatment failed to inhibit neutrophilic airway inflammation, and sometimes even aggravated the pathologies. When the IL-17A/DEX synergy was blocked by IL-17A inhibition or CSF3 neutralization, DEX sensitivity was acquired in these neutrophilic airway disease models. Interestingly, we also found that cyanidin-3-glucoside (C3G), a small-molecule inhibitor that blocks IL-17A binding to IL-17RA, when used in combination with DEX, could improve DEX sensitivity and effectively inhibit neutrophilic inflammation and associated pathological features in the preclinical models of severe asthma and COPD, offering a promising therapeutic approach for treating these diseases.

\section{Results}

GC differentially regulates IL-17A-induced neutrophil-promoting cytokines in ASMCs. To investigate the mechanisms of steroid resistance in IL-17A-mediated neutrophilic airway diseases, we performed RNA sequencing (RNA-seq) analysis of transcriptomes in IL-17A- and DEX- treated human ASMCs (Figure 1A). Two groups of IL-17A target genes were identified showing differential responses to DEX treatment: steroid sensitive and steroid resistant. Interestingly, the expression of those genes (e.g., CSF2, CXCL1, CXCL2, IL6, and IL8), well known to promote neutrophil functions, was effectively inhibited by DEX treatment with or without IL-17A. In contrast, DEX induced CSF3 expression alone, although the induction level was not high. Interestingly, DEX-induced CSF3 expression was dramatically increased in the presence of IL-17A. The IL-17A/ DEX-mediated synergistic induction of CSF3 mRNA was validated by real-time PCR analysis of mRNA transcripts in both human and mouse ASMCs (Figure 1B and Supplemental Figure 1A; supplemental material available online with this article; https://doi.org/10.1172/jci.insight.132836DS1). Notably, IL-17A alone is a relatively weak inducer of inflammatory gene expression. Previous studies have demonstrated that the pathogenic role of IL-17A is executed through its synergic cooperation with other inflammatory cytokines, including TNF (16). Importantly, DEX was able to further promote IL-17A/TNF-induced CSF3 expression in a synergistic manner, whereas DEX effectively suppressed the expression of CSF2, CXCL1, and CXCL2 (Figure 1B and Supplemental Figure 1A). We also observed similar gene induction patterns for Csf2, Cxcl1, $C x c l 2$, and $C s f 3$ in response to IL-17A and DEX in mouse lung fibroblasts, suggesting that similar gene regulation mechanisms may operate in multiple cell types (Supplemental Figure 1B).

To understand the molecular mechanisms involved in cooperative induction of CSF3 expression by IL-17A and DEX, we performed promoter analysis of human CSF3 in A594 cells (an IL-17A-responsive human airway epithelial cell line). WT (-924/+89) human CSF3 promoter luciferase plasmid was transfected into A594 cells, followed by IL-17A and DEX treatment, alone or in combination. As shown in Figure 1C, IL-17A and DEX induced the CSF3 promoter (WT, -924/+89) only in an additive manner, suggesting that a posttranscriptional mechanism exists for the synergy between IL-17A and DEX. Thus, we performed a CSF3 mRNA decay assay using actinomycin D (a transcription inhibitor) following DEX pretreatment. CSF3 mRNA decayed at a slower rate in the presence of IL-17A as compared with that without IL-17A (Figure 1D), indicating that IL-17A signaling enhanced stability of CSF3 mRNA induced by DEX. In contrast, DEX was unable to stabilize either basal or IL-17A-induced CSF3 mRNA (without DEX pretreatment) (Supplemental Figure 2, A and B). GCs signal via the GC receptor (GR, encoded by NR3C1) to exert their transcriptional activation and repression activities by binding GC response elements (GREs). Using JASPAR (an open-access database of curated, nonredundant transcription factor binding profiles; http://jaspar. genereg.net) (17), we identified putative binding sites for multiple transcription factors (including NF- $\mathrm{B}$, $\mathrm{NR} 3 \mathrm{C} 1$, and $\mathrm{C} / \mathrm{EBP}$ ) in the human CSF3 promoter. Two overlapping GRE sequences (TGGGACAGGACAGCCTG and CAGGACAGCCTGGAACT) (Figure 1E) were predicted using an NR3C1 binding sequence logo (generated by JASPAR) (Figure 1F) in the NR3C1a region, which is highly conserved between human and mouse. Although deletion of sequences containing either NF-kB1 [Mut1(-589/+98), Del-NF-

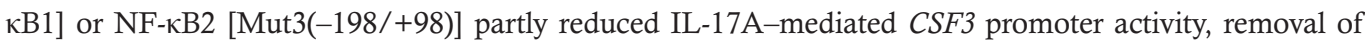
sequences containing NR3C1a [Mut1(-598/+89), Mut2(-538/+89), Mut3(+198/+89), Mut4(+148/+89), Del-NR3C1a] completely abolished DEX-induced CSF3 promoter activity, indicating that NR3C1a is a true GRE-containing region in the CSF3 promoter (Figure 1G). Similar to what we observed for IL-17A, NR3C1a deletion had little effect on TNF-induced CSF3 promoter activity, but removal of the first $2 \mathrm{NF}-\mathrm{\kappa B}$ sites almost completely abolished TNF-mediated promoter activity. Notably, TNF had a stronger effect on CSF3 promoter activity as compared with IL-17A or DEX but was unable to stabilize CSF3 mRNA with or without IL-17A (Supplemental Figure 2, A and B). We also performed a ChIP assay to investigate if DEX could induce GR binding to the NR3C1a region in the human CSF3 promoter (Figure $1 \mathrm{H}$ ). 
A

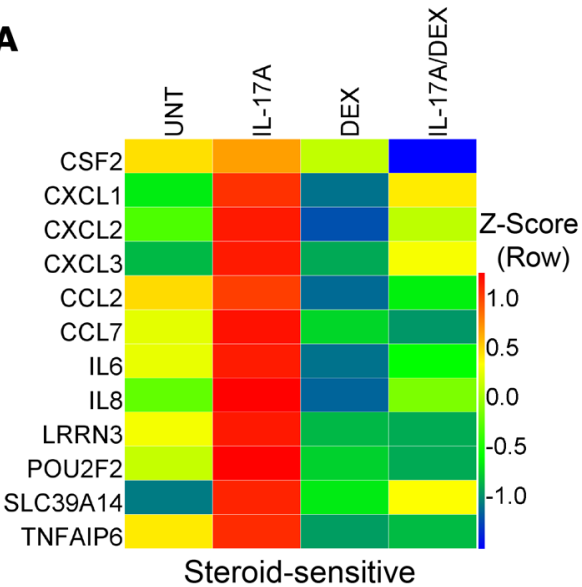

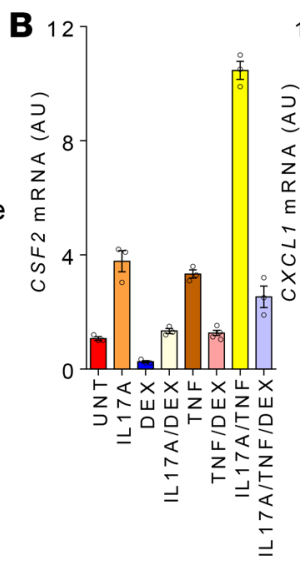

C

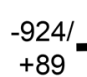

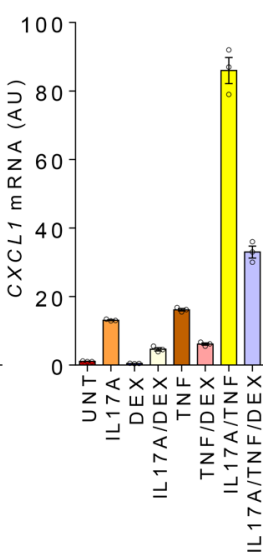

TSLuc

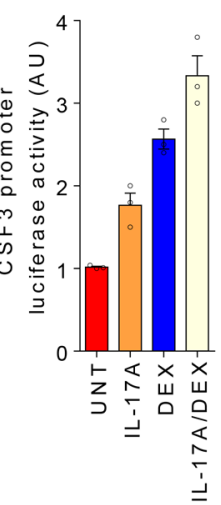

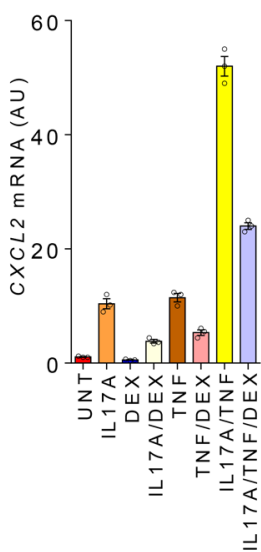

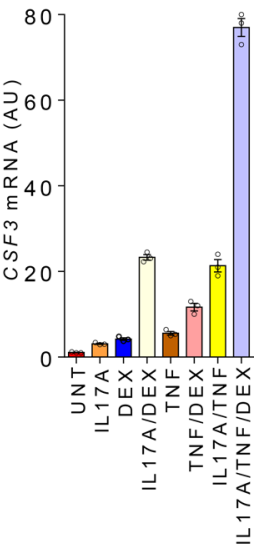

D

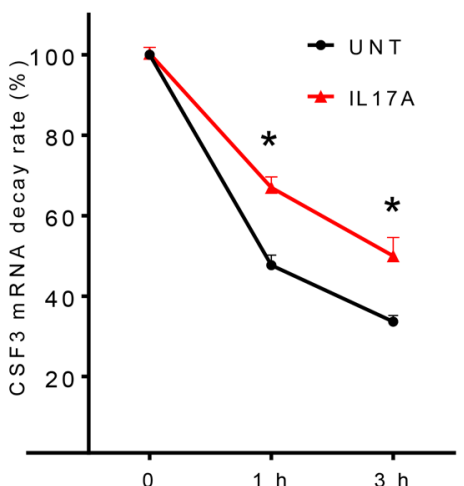

E

Steroid-resistant

CEBPD Oorf10 MAP3K8

MMP15 NFKBID SAA1 STEAP4 ZC3H12A ZC3H12C

Steroid-resistant

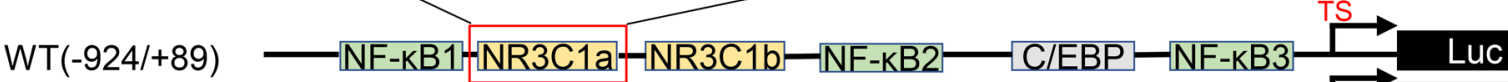

Mut1(-589/+89)

Mut2(-538/+89)

Mut3(-198/+89)

Mu4(-148/+89)

D-NR3C1a

D-NFkB1

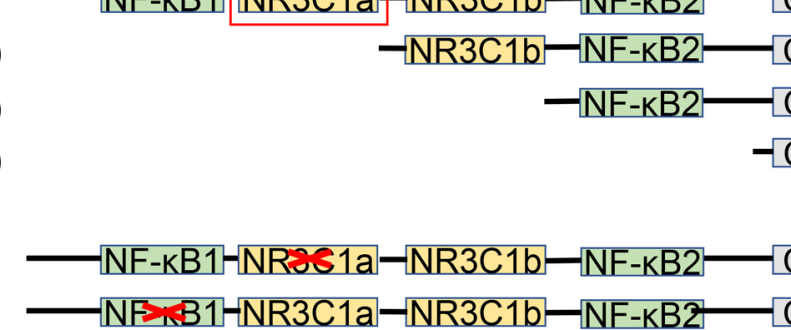

G

\begin{tabular}{|c|c|c|c|c|c|c|c|}
\hline & & & $\begin{array}{l}\text { IL17A } \\
\text { /DEX }\end{array}$ & & $\begin{array}{l}\text { IL17A } \\
\text { /TNF }\end{array}$ & $\begin{array}{l}\text { TNF } \\
\text { /DEX }\end{array}$ & $\begin{array}{c}\text { IL17A } \\
\text { /TNF/DEX }\end{array}$ \\
\hline WT(-924/+89) & $1.9 \pm 0.1$ & & $3.5 \pm 0.2$ & $3.5 \pm 0.1$ & $5.4 \pm 0.1$ & $9.8 \pm 0.2$ & $14.8 \pm 0.3$ \\
\hline Mut 1 & & & & & & & \\
\hline & & & $1.5 \pm 0.1$ & & & & \\
\hline & $1.1 \pm 0.1$ & & $1.1 \pm 0.1$ & 0.1 & & $1 \pm 0.1$ & \\
\hline Mut4 & $1.0 \pm 0.0$ & & $1.0 \pm 0.1$ & $1.0 \pm 0.1$ & $1.0 \pm 0.1$ & & \\
\hline & $1.5 \pm 0.1$ & & $1.6 \pm$ & $3.4 \pm$ & 4.2 & & 1 \\
\hline el- & $1.4 \pm 0.1$ & $2.5 \pm 0.1$ & $3.2 \pm 0.1$ & $2.5 \pm 0.1$ & $4.0 \pm 0.1$ & $2.2 \pm 0.1$ & $7.1 \pm 0.2$ \\
\hline
\end{tabular}

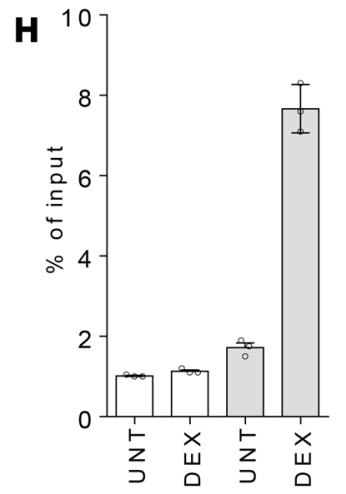

IP: $\overline{\lg G}$ anti-GR 
Figure 1. Glucocorticoid differentially regulates IL-17A-induced neutrophil-promoting cytokines in ASMCs. (A) RNA-seq analysis of human ASMCs (hASMCs) untreated (UNT) and treated for 4 hours with IL-17A (100 ng/mL), DEX $(1 \mu \mathrm{M})$, or both. Shown are representative DEX-sensitive and DEX-resistant genes. $Z$ scores were calculated as $\log (F P K M)$ values. (B) Real-time PCR analysis of mRNA expression of indicated cytokines in hASMCs treated as indicated in A. (C) WT human CSF3 promoter luciferase plasmids were transfected into A549 cells. After 24 hours, the cells were treated as indicated in $\mathbf{A}$ for 6 hours, followed by luciferase assay. The luciferase activity is expressed as fold induction relative to untreated control. (D) mRNA degradation assay. hASMCs were pretreated with DEX for 4 hours, followed by treatment with actinomycin $D(5 \mu \mathrm{g} / \mathrm{mL})$ in the absence and presence of IL-17A (100 ng/mL). mRNA decay rate was measured as a ratio (percentage) of remaining mRNA at indicated time points to the amount of mRNA at time point 0 . Data represent mean \pm SEM ( $n=3$ biological replicates). ${ }^{*} P<0.05$ (2-tailed Student's $t$ test). (E) Schematic representation of the WT and mutated human CSF3 luciferase reporter constructs. (F) NR3C1 binding sequence logo. (C) WT or mutant human CSF3 luciferase constructs were transfected into A549 cells, followed by luciferase assay as indicated in C. (H) ChIP analysis of DEX-induced binding of GR to the human CSF3 promoter. Nucleic extracts from untreated and treated hASMCs were immunoprecipitated with IgG or anti-GR, followed by DNA purification and RT-PCR quantitation with primers spanning the putative NR3C1 binding site of the CSF3 promoter. TS, transcription start site; GR, glucocorticoid receptor. For panels B, C, G, and $\mathbf{H}$, data represent mean \pm SD ( $n=3$ technical replicates). All data are representative of 3 independent experiments.

Using primer pairs spanning NR3C1a, we confirmed that DEX indeed induced GR binding to the NR3C1a region in a synergistic manner. The above results indicate that DEX differentially regulates IL-17A-induced neutrophil-promoting genes and synergistic induction of CSF3 expression by IL-17A and DEX involves both transcriptional and posttranscriptional mechanisms.

Disruption of IL-17A/DEX synergistic effect on Csf3 expression renders DEX sensitivity in HDM-CFA type17 acute asthma model. House dust mite (HDM) is a natural allergen to which asthmatics are commonly exposed. By using different immunization adjuvants (alum and CFA), we established HDM-induced steroid-sensitive type-2 (Th2-high) and steroid-resistant type-17 (Th17-high) mouse acute asthma models, which respectively recapitulate pathological features of eosinophilic mild/moderate asthma and neutrophilic severe asthma in human patients (Supplemental Figure 3) (18). In the type-2 model (induced by HDM-alum, Supplemental Figure 3, A-D), airway inflammation was characterized by predominant infiltrating eosinophils ( $>70 \%$ in bronchoalveolar lavage [BAL]), type-2 cytokine gene induction (Ill7e, Il4, Il5, and Il13), and sensitivity to low-dose DEX $(0.3 \mathrm{mg} / \mathrm{kg} \mathrm{BW})$ treatment. In contrast, the type-17 model (induced by HDM-CFA; Supplemental Figure 3, E-H) featured prevalent neutrophilic inflammation ( $>80 \%$ neutrophils in BAL), and upregulated IL-17A and neutrophil-promoting genes (Csf3, Cxcl1, and Cxcl2). Interestingly, although DEX $(0.3 \mathrm{mg} / \mathrm{kg} \mathrm{BW})$ treatment was able to reduce the expression of $C x c 11$ and $C x c l 2$, it increased Csf3 expression and failed to inhibit neutrophilia in the airway. Consistently, in DEX-treated mice on the HDM-CFA model, the CSF3 level but not the CXCL1 level in lung tissue was correlated with the number of neutrophils in the BAL, suggesting a pivotal role of CSF3 in the DEX-resistant neutrophilic airway inflammation (Supplemental Figure 4, A and B).

We then investigated the physiological implications of synergistic induction of Csf 3 expression by IL-17A and DEX in vivo in mice. Considering the observation on the synergistic effect between IL-17A and DEX on the neutrophil-promoting gene CSF3 in ASMCs, we aimed to examine the impact of the IL-17A/DEX/CSF3 axis on airway neutrophilic inflammation. Using the HDM-CFA type-17 acute asthma model (Supplemental Figure 3E), we found that systemic delivery of anti-IL-17A neutralizing antibody to mice reduced neutrophilic inflammation in the airway and improved DEX sensitivity, as indicated by BAL cell counts (Figure 2A). This is consistent with the expression profile of neutrophil-promoting genes in lung tissue; while anti-IL-17A alone reduced the expression of Csf3 as well as Cxcl1 and Cxcl2, DEX treatment in the presence of anti-IL-17A further diminished the expression of $C x c l 1$ and $C x c l 2$ and did not enhance $C s f 3$ expression, as observed in mice on DEX treatment alone (Figure 2B). Like Cxcl1 and Cxcl2, other steroid-sensitive IL-17A target genes (Il6, Il8, and Tnf) showed a similar induction pattern upon DEX and/or anti-IL-17A treatments (Supplemental Figure 5). We then wondered whether ablating CSF3 would be sufficient to recuperate DEX sensitivity for neutrophilic inflammation in this HDM-CFA model. We indeed observed that anti-CSF3 neutralizing antibody, like anti-IL-17A, decreased airway neutrophilia/ neutrophil-promoting gene expression, rendering DEX sensitivity in treated mice (Figure 2, A and B, and Supplemental Figure 5). Taken together, these results show that DEX alone, albeit failing to inhibit airway neutrophilic inflammation, was able to do so in the presence of anti-IL-17A or anti-CSF3. The above results suggest that the IL-17A/DEX/CSF3 axis plays a critical role in the pathogenesis of steroid-resistant neutrophilic airway inflammation. Therefore, disrupting the IL-17A/DEX synergistic effect on CSF3 expression might be an attractive, new steroid-sparing therapeutic approach for treating neutrophilic airway diseases.

Small-molecule IL-17A blocker C3G alleviates neutrophilia and improves DEX sensitivity in HDM-CFA type-17 acute asthma model. Owing to the considerable cost of antibody production and administration limitation 
A

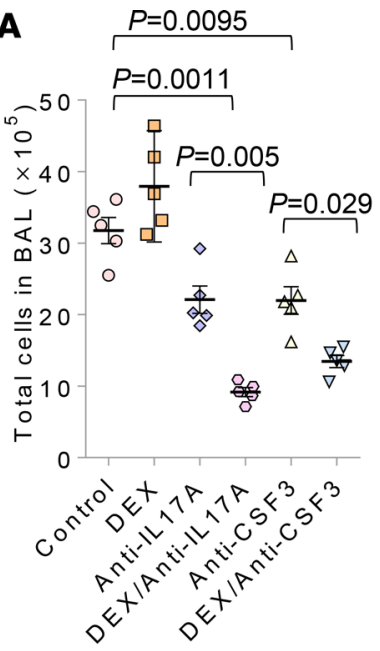

B

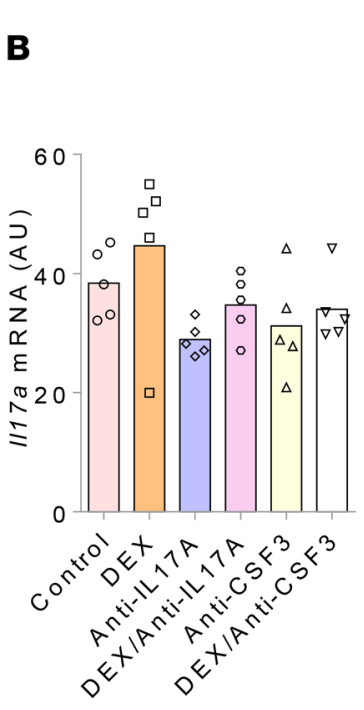

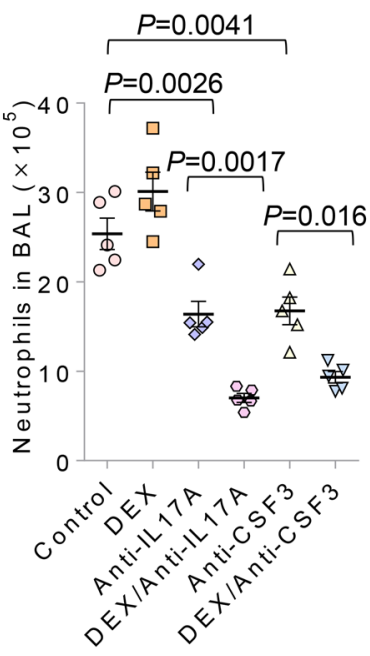

Figure 2. Disruption of IL-17A/DEX synergistic effect on CSF3 expression renders DEX sensitivity in HDM-CFA type-17 acute asthma model. (A) Eight-week-old WT C57BL/6 female mice ( $n=5$ per group) were subjected to the HDM-CFA acute severe asthma model. Isotype antibody (Control), DEX, anti-CSF3, and anti-IL-17A neutralizing antibodies or combined DEX and antibodies were administered to the mice (as described in Methods). Twenty-four hours after challenging, total cell and neutrophil counts in the BAL were quantified. (B) mRNA expression of lung tissues was quantified by real-time PCR. AU, fold induction relative to unchallenged control mice. Data represent mean \pm SEM. One-way ANOVA was performed, followed by Tukey's multiple-comparisons test. The multiplicity-adjusted $P$ values were calculated for the indicated comparisons. The above data are representative of 3 independent experiments.
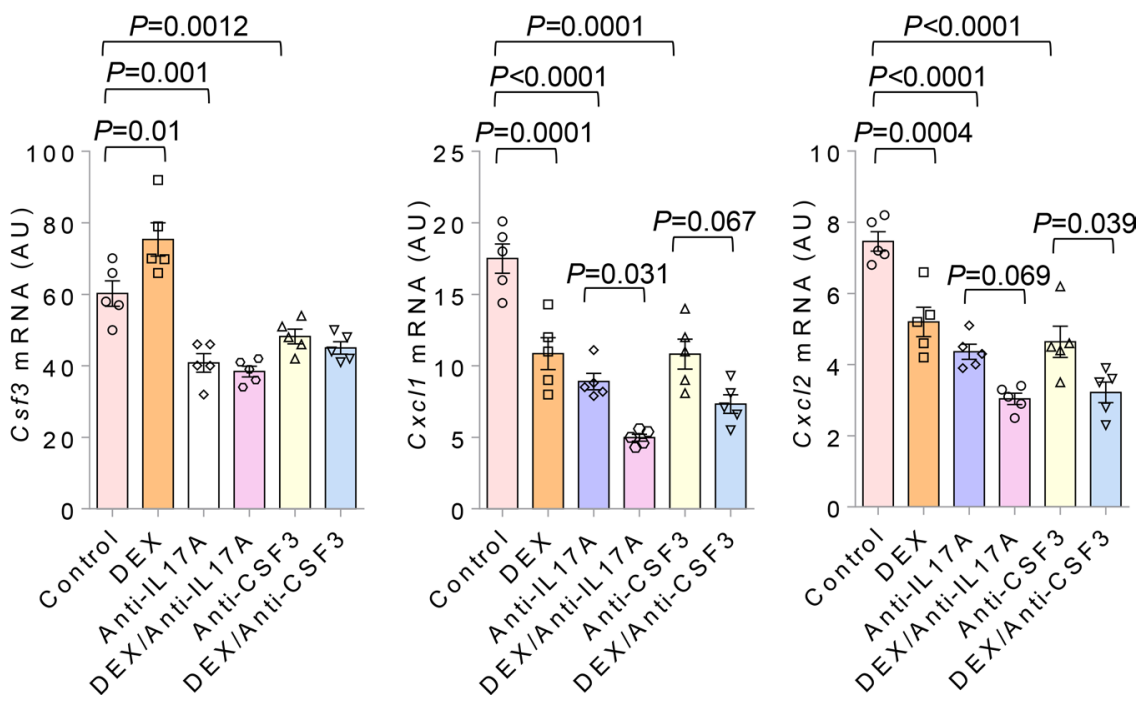

(only through the intravenous route), we sought to investigate small-molecule inhibitors to block IL-17A/ DEX synergy. We have previously identified a small-molecule natural compound (cyanidin) that can effectively block IL-17A binding to its receptor IL17RA via computer-based virtual screening (18). Interestingly, C3G showed similar bioactivity to that of cyanidin in cell-base assays (Figure 3, A and B). Because C3G has been safely used in human clinical studies $(19,20)$, we sought to pursue it as a small-molecule inhibitor to block IL-17A/DEX synergy. We evaluated the ability of C3G alone or in combination with DEX in alleviating neutrophilic airway inflammation in the neutrophilic HDM-CFA type-17 acute asthma model. As shown in Figure 3, C-E, and Supplemental Figure 6, HDM-CFA-induced airway neutrophilic inflammation (as indicated by BAL count and lung histology) and the expression of neutrophil-promoting genes (Csf3, Cxcl1, Cxcl2, Il6, Il8, and Tnf) in the lung tissue were reduced by C3G treatment alone. Interestingly, DEX/C3G combined treatment showed a much more dramatic reduction in neutrophilic inflammation as compared with $\mathrm{C} 3 \mathrm{G}$ single treatment. In support of this, the expression of steroid-sensitive cytokines (Csf3, Cxcl1, Cxcl2, Il6, Il8, and Tnf) were greatly reduced in the lung tissue of DEX/C3G-treated mice as compared with that in mice treated with $\mathrm{C} 3 \mathrm{G}$ only, while $C S f 3$ expression was not enhanced in the presence of $\mathrm{C} 3 \mathrm{G}$ as compared with that in mice treated with DEX alone.

To further validate the in vivo bioactivities of C3G for alleviating neutrophilic airway inflammation, we tested C3G in a neutrophilic Th17 adoptive transfer asthma model that was IL-17A driven and steroid resistant (13). In this model, like what we observed in the HDM-CFA type-17 acute asthma model, DEX exhibited a trend to worsen neutrophilic airway inflammation (Figure 3, F and G). However, even though C3G alone alleviated the inflammation, the therapeutic effect was much more dramatic when used in combination with DEX. The above results indicate that $C 3 G$ is an effective 
A<smiles>Oc1cc(O)c2cc(OC(Cl)(Cl)Cl)c(-c3ccc(O)c(O)c3)[o+]c2c1</smiles>

B

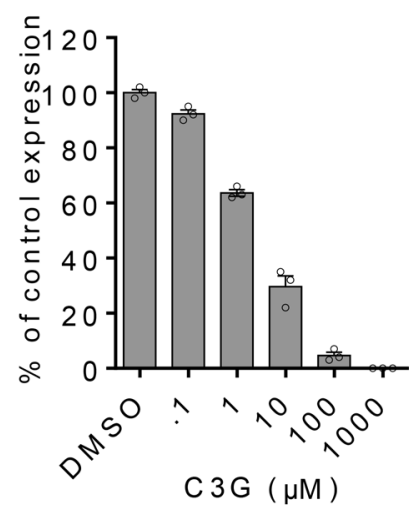
C $3 G(\mu M)$ m ASMC

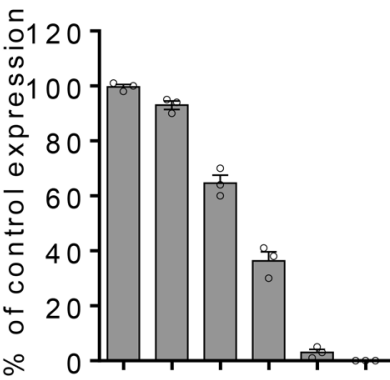

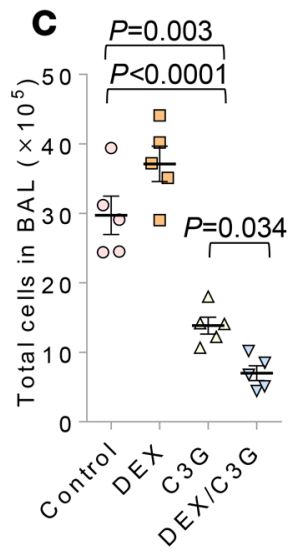

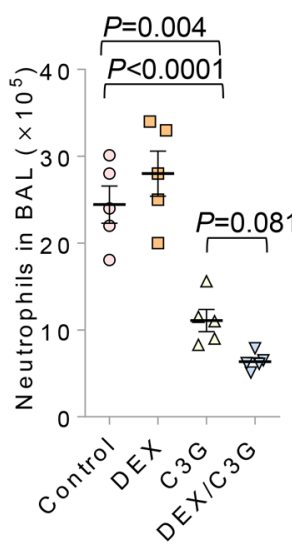

D
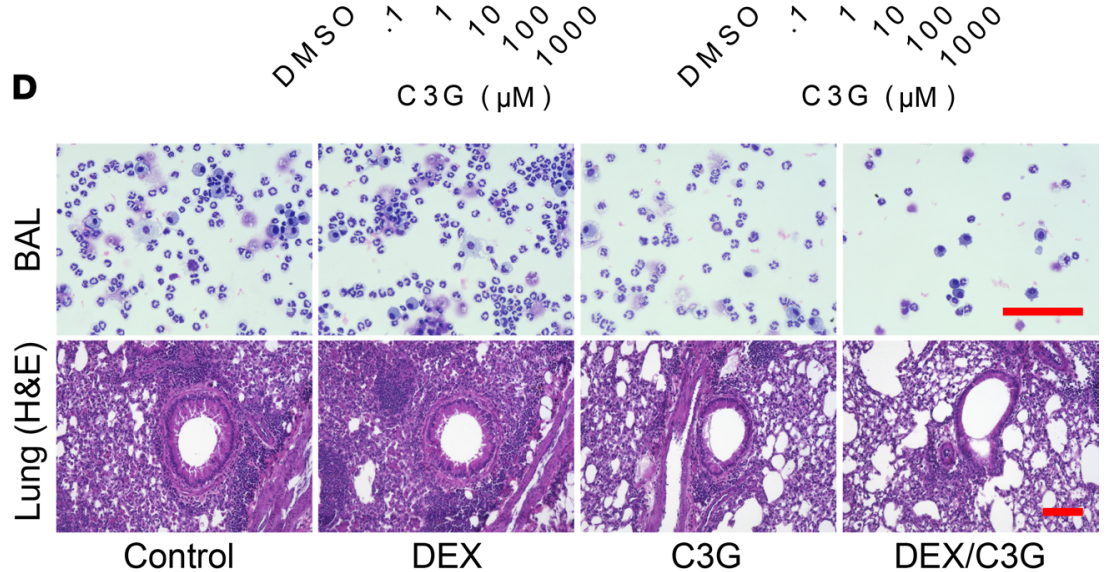

$\mathbf{E}$

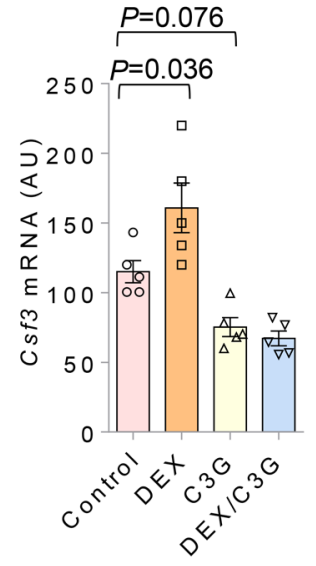

$\mathbf{F}$
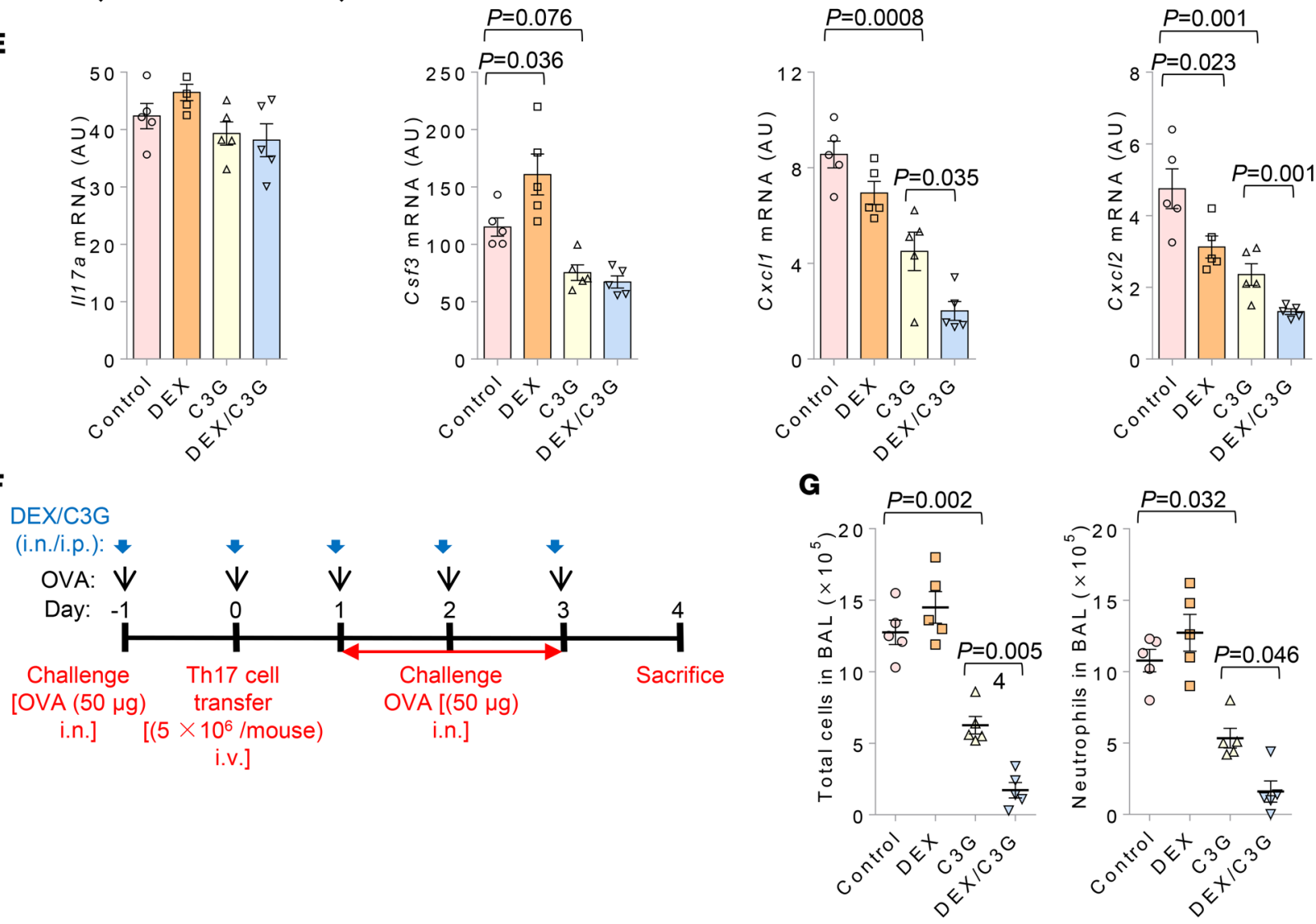

Figure 3. Small-molecule IL-17A blocker C3G alleviates neutrophilia and improves DEX sensitivity in HDM-CFA type-17 acute asthma model. (A) Chemical structure of C3G. (B) Human and mouse ASMCs (hASMCs and mASMCs) were treated for 24 hours with IL-17A in the presence of DMSO (vehicle control) or the indicated concentrations of C3G. The concentrations of CXCL1 (GRO $\alpha$ in humans and KC in mice) in the culture medium were measured by ELISA. Results are presented as a percentage of the amount of CXCL1 in the culture medium of DMSO-treated cells, which was set at 100\%. Data are representative of 3 independent experiments. Error bars represent the SEM of technical replicates. (C and D) Eight-week-old WT C57BL/6 female mice ( $n=5$ per group) were 
subjected to the HDM-CFA acute asthma model. PBS (Control), DEX, C3G, or a combination of DEX and C3G were administered to the mice (as described in Methods). Twenty-four hours after challenging, total and neutrophil counts in the BAL were quantified (C). Representative BAL cells were prepared by cytospin and lung tissues were stained with H\&E (D). All scale bars (red): $100 \mu \mathrm{m}$. (E) mRNA expression of lung tissues was quantified by real-time PCR. (F and G) $\mathrm{OVA}_{323-339}$-specific Th17 cells were adoptively transferred into 8-week-old female C57BL/6 mice ( $n=5$ mice per treatment group), which were treated as described in Methods. Twenty-four hours after the last challenge, total cell and neutrophil counts in the BAL were quantified. AU, fold induction relative to unchallenged control mice. Data represent mean \pm SEM. One-way ANOVA was performed, followed by Tukey's multiple-comparisons test. The multiplicity-adjusted $P$ values were calculated for the indicated comparisons. The above data are representative of 3 independent experiments.

small-molecule alternative for anti-IL-17A/IL-17RA antibodies and was able to render DEX sensitivity in IL-17A-mediated neutrophilic asthma. Combined used of DEX and C3G can serve as an effective approach to treat steroid-resistant neutrophilic airway diseases.

C3G ameliorates airway remodeling and mitigates DEX resistance in HDM-CFA type-17 chronic asthma model. Given the excellent efficacy of DEX/C3G combination in blocking the expression of neutrophil-promoting genes and airway neutrophilia, it is warranted to further examine the potential therapeutic effect in preclinical models with more relevant pathologies to human asthma. The major features of asthma in chronic phase are irreversible airway structural changes (also called airway remodeling) as manifested by epithelial alteration, goblet cell hyperplasia, ASM hypertrophy/hyperplasia, and fibrosis. IL-17A has been shown to play an important role in these pathological features (21-25). We established an HDM-CFA type17 chronic asthma model that exhibited persistent neutrophilic airway inflammation, increased collagen deposition, and upregulated expression of inflammatory (III7, Csf3, Cxcl1, and Cxcl2) and remodeling genes (Tgfb1, Muc5b, Colla1, and Acta2), many of which were greatly alleviated upon depletion of IL-17A and neutrophils with neutralizing antibodies (Figure 4A and Supplemental Figure 7). We treated the mice with DEX and C3G, alone or in combination, during the challenge stage of this chronic model (Figure 4A). As shown in Figure 4, B-E, we found that C3G alone was effective in inhibiting neutrophilic inflammation (as indicated by BAL count and lung histology) and airway remodeling (as indicated by increased mucus production detected using periodic acid-Schiff [PAS] staining), collagen deposition (trichrome staining and total collagen in lung tissue), and SMC layer thickening (smooth muscle actin [SMA] staining). The expression of inflammatory and remodeling genes (Csf3,Cxcl1, Cxcl2, Tgfb1, Muc5b, Colla1, and Acta2) was also reduced in lung tissue by C3G treatment (Figure $4 \mathrm{~F}$ ). DEX alone promoted neutrophilic inflammation but did not affect airway remodeling, suggesting the existence of neutrophil-independent remodeling pathways. DEX reduced $C x c l 1$ and $C x c l 2$ expression but significantly enhanced $C s f 3$ expression in lung tissue. The combined treatment with $\mathrm{C} 3 \mathrm{G}$ and DEX is more efficacious for alleviating inflammation and remodeling as compared with C3G alone, suggesting that C3G indeed improved DEX sensitivity by blocking IL-17A/ DEX synergy to prevent airway remodeling associated with neutrophilic chronic asthma.

$C 3 G$ reduces emphysema and restores $D E X$ sensitivity in elastase-induced neutrophilic COPD. We then sought to examine the potential therapeutic efficacy of DEX/C3G combination in another neutrophilic airway disease model. COPD is a small-airway disease characterized by neutrophilic inflammation, emphysema (enlarged air space size due to disrupted alveoli), increased mucus production, smooth muscle hyperplasia and hypertrophy, and fibrosis. Neutrophilic COPD is resistant to steroid-based therapy, which usually has no effect on preventing disease progression. IL-17A has been strongly implicated in the pathogenesis of COPD. IL-17A expression is elevated in COPD patients $(4,26)$. Genetic ablation of IL-17A signaling components or anti-IL-17A treatment can greatly alleviate pathological features associated with COPD in mouse models $(3,8,27)$. In the current study, we used an elastase-induced COPD model to test the efficacy of $C 3 \mathrm{G}$ and combined treatment of C3G and DEX (Figure 5A). Like what we observed in the HDM-CFA chronic asthma model, while DEX treatment alone did not exhibit any beneficial effect, C3G was able to reduce the key pathological features of COPD, including airway neutrophilic inflammation and emphysema (Figure 5, B-D). It is important to note that DEX itself substantially increased Csf3 expression in lung tissue while it reduced the expression $\mathrm{Cxcl}$ and $\mathrm{Cxcl2}$ (Figure 5E). Combined DEX/ C3G treatment had better efficacy to alleviate COPD symptoms than C3G single treatment, suggesting that $\mathrm{C} 3 \mathrm{G}$ also improved DEX sensitivity in COPD. Consistently, DEX/C3G combination, as compared with C3G treatment, further reduced the expression of inflammatory genes ( $\mathrm{CxCl1}$ and $\mathrm{Cxcl2}$ ) and remodeling genes (Tgfb1, Muc5b, Colla1, and Acta2) and total collagen content in lung tissue (Figure 5, E and F).

CSF3 induced by IL-17A/DEX in ASMCS is the key neutrophil survival cytokine. Because the results from our in vitro cell culture experiments suggest that ASMCs can be an important source of neutrophil-promoting 
A

DEX/C3G

(i.n./i.p.):

HDM:

Week:

Sensitization [HDM $(100 \mu \mathrm{g}$

in CFA s.c.]
[HDM $(25 \mu g)$ i.n., twice per week]

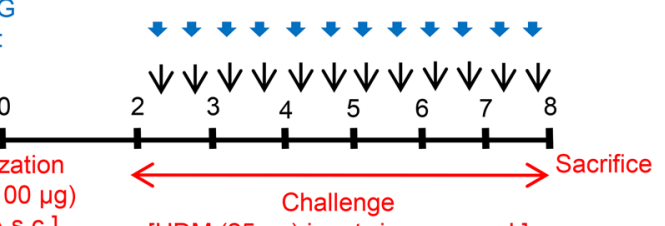

C

\section{㝒}

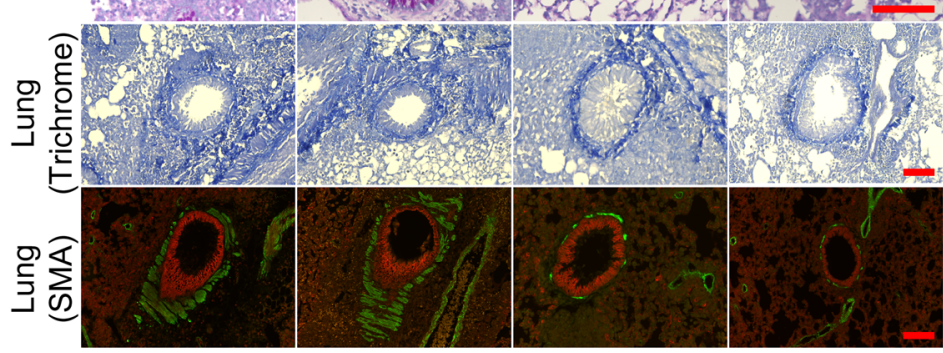

Control

$\mathbf{F}$
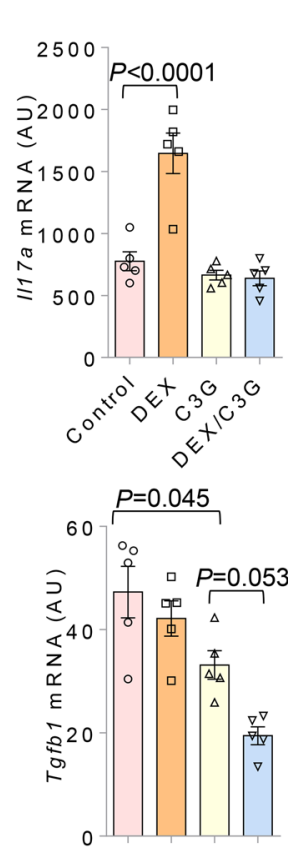

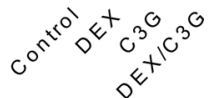

DEX
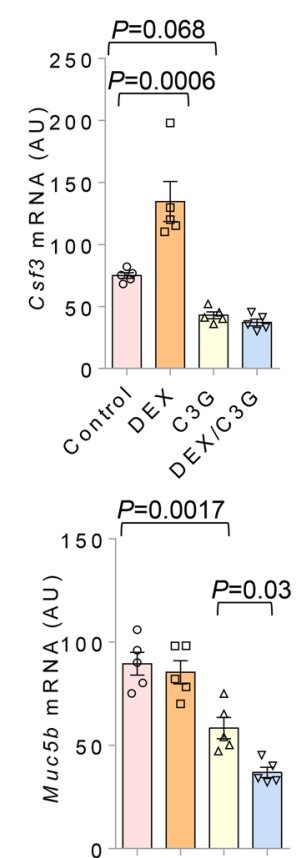

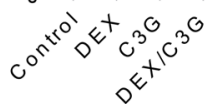

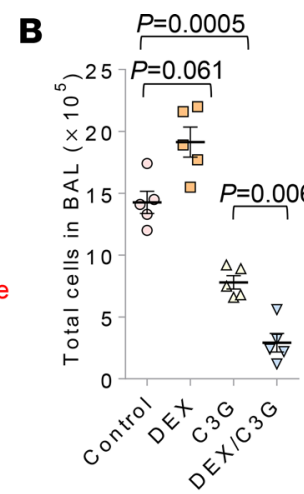

D
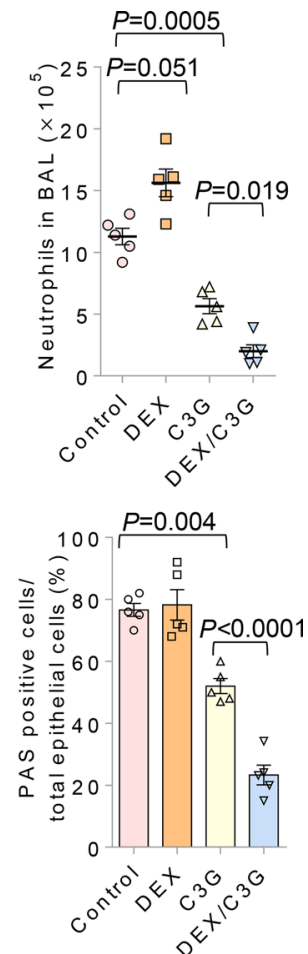

E

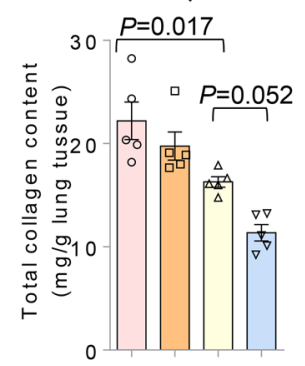

E-cadherin
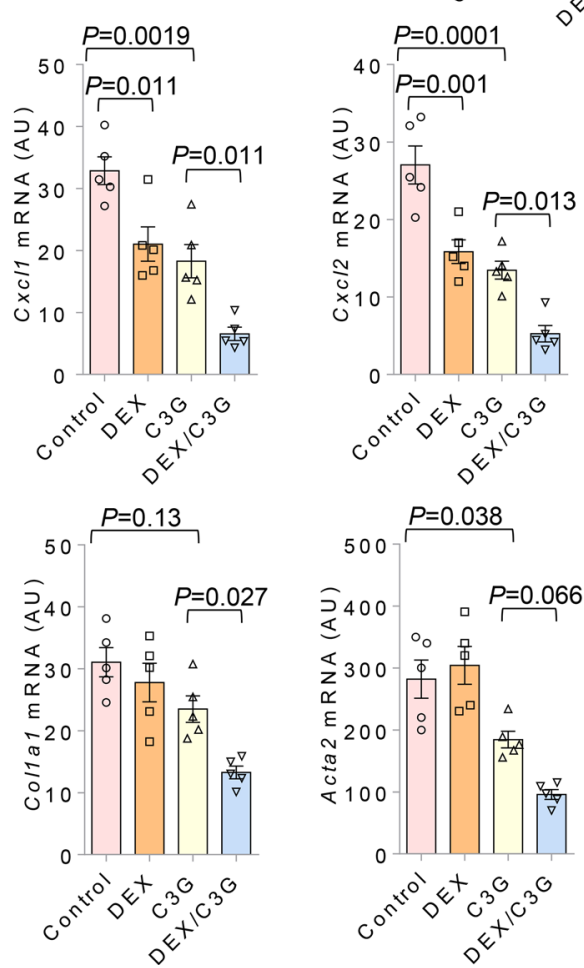
Figure 4. C3G ameliorates airway remodeling and mitigates DEX resistance in the HDM-CFA type-17 chronic asthma model. (A) Eight-week-old WT C57BL/6 female mice ( $n=5$ mice per group) were subjected to the HDM-CFA chronic asthma model. PBS (Control), DEX, C3G, or a combination of DEX and C3G were administered to the mice (as described in Methods). Twenty-four hours after the last challenge, total cell and neutrophil counts in the BAL were quantified (B), and representative BAL cells were prepared by cytospin and lung tissues were subjected to histochemical staining as indicated (C). All scale bars (red): $100 \mu \mathrm{m}$. (D and E) PAS score (PAS-positive cells/total epithelial cells) and total collagen content of lung tissue were measured. (F) mRNA expression of lung tissues was quantified by real-time PCR. AU, fold induction relative to unchallenged control mice. Data represent mean \pm SEM. One-way ANOVA was performed, followed by Tukey's multiple-comparisons test. The multiplicity-adjusted $P$ values were calculated for the indicated comparisons. The above data are representative of 3 independent experiments.

cytokines (including CXCL1, CXCL2, and CSF3) upon IL-17A stimulation, we examined the impact of cell-specific deletion of IL-17A signaling in ASMCs on airway neutrophilic inflammation in the HDMCFA type-17 acute asthma model. We bred $I l 17 r c$ (IL-17A receptor subunit) floxed mice (Il17r $\left.{ }^{\mathrm{f} / \mathrm{fl}}\right)$ with SMA-rtTA-Cre transgenic mice [expressing the reverse tetracycline transactivator under the control of the $\alpha$-SMA (encoded by Acta2) promoter ( $\alpha$-SMA-rTTA) and (tetO)7-cre] to generate SMA-specific (Acta2-specific) $I l 17 r c$-deficient mice (SMA-Il17rct/fl $)$ and control mice (SMA-Il17rct/++) (28). As shown in Figure 6, A and B, SMA-Il17rct/fl mice showed greatly reduced airway neutrophilic inflammation as indicated by BAL count, lung histology, and reduced expression of neutrophil-promoting genes (Csf,$C x c l 1$, and $C x c l 2)$ in lung tissue in the HDM-CFA model as compared with control mice (SMA-Ill7 $r c^{\mathrm{Al} /+}$ ), indicating that IL-17A signaling in ASMCs is critical in the pathogenesis of type-17 severe asthma. We also performed a flow cytometry-based TUNEL assay for lung neutrophils isolated from SMA-Il17rct/fli and control SMA-Il17rct/+ mice subjected to the HDM-CFA type 17 acute asthma model (Supplemental Figure 8 and Figure 6C). The results showed that the lung neutrophils (gated as $\mathrm{CD} 45^{+} \mathrm{CD} 11 \mathrm{~b}^{+} \mathrm{Ly} 6 \mathrm{G}^{+}$) in control SMA-Ill7rct/+ mice had a lower apoptotic rate as compared with SMA- $I l 17 r c^{\mathrm{fl} / \mathrm{fl}}$ mice, suggesting that neutrophil survival was affected by ablating IL-17A signaling in ASMCs.

To address the critical role of CSF3 induced by IL-17A and DEX in ASMCs in neutrophilic airway inflammation, we performed survival experiments using neutrophils isolated from mouse BM. We cultured BM-derived neutrophils with conditioned medium harvested from mouse ASMCs treated with IL-17A and DEX, alone or in combination. As DEX is known to promote neutrophil survival itself (29), we used a GR-specific inhibitor (AL082D06) (30) to block the direct effect of DEX on neutrophils in the conditioned medium. As shown in Figure 6D, we found that conditioned media from mouse ASMCs treated with IL-17A and DEX alone increased the neutrophil survival rate, and IL-17A/DEX combined treatment conferred a synergistic effect. This was consistent with the Csf3 gene induction pattern observed in mouse ASMCs treated with IL-17A and DEX (Supplemental Figure 1A). C3G only blocked the IL-17A treatment effect. However, CSF3-neutralizing antibody completely abolished the survival-promoting effect of all conditioned media, suggesting that CSF3 is the sole critical survival factor in these conditioned media. By contrast, only media from IL-17A-treated mouse ASMCs could induce neutrophil migration in Transwell migration assays, suggesting that IL-17A but not DEX induced neutrophil-migrating chemokines (e.g., CXCL1 and CXCL2) (Figure 6E). Both DEX and C3G treatments reduced IL-17A-induced migratory factors from mouse ASMCs and combined treatment completely abolished the migratory effect from the media of IL-17A-treated mouse ASMCs.

\section{Discussion}

IL-17A is an important contributing factor in the pathogenesis of neutrophilic airway diseases including severe asthma and COPD (4). IL-17A induces production of an array of neutrophil-promoting cytokines/ chemokines (e.g., CSF2, CXCL1, CXCL2, CSF3, IL-6, and IL-8) to elicit airway neutrophilic inflammation. Asthma and COPD are commonly treated by GC-based antiinflammatory agents. However, although type-2 eosinophilic inflammation can be effectively inhibited by GC-based therapy, neutrophilic inflammation is resistant to GC treatment and often worsened by GC treatment under certain circumstances $(3,6-10)$. Moreover, high-dose use of steroid drugs has been associated with many metabolic side effects (31-33). Therefore, there is an unmet clinical demand to develop GC-sparing agents or alternative therapeutics for treating neutrophilic airway diseases. Understanding the underlying mechanisms of steroid resistance will provide important guidance for developing therapeutic strategies. In the present study, we uncovered what we believe is a novel mechanism of steroid resistance in neutrophilic airway diseases. We found that DEX induced neutrophil-promoting CSF3 expression in synergy with IL-17A in ASMCs, the mechanisms of which involved cooperative regulation at both transcriptional and posttranscriptional levels. 
A

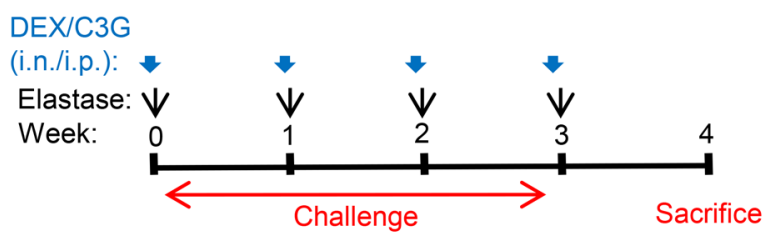

(Elastase $(0.3 \mu \mathrm{g} /$ mouse, i.n.)

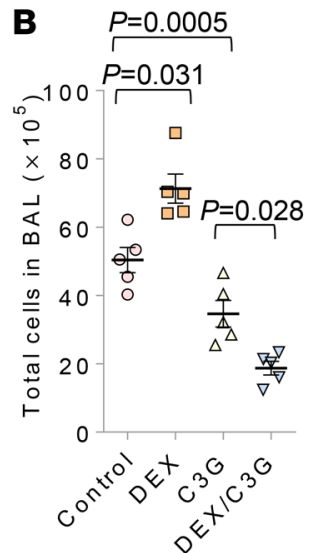

C
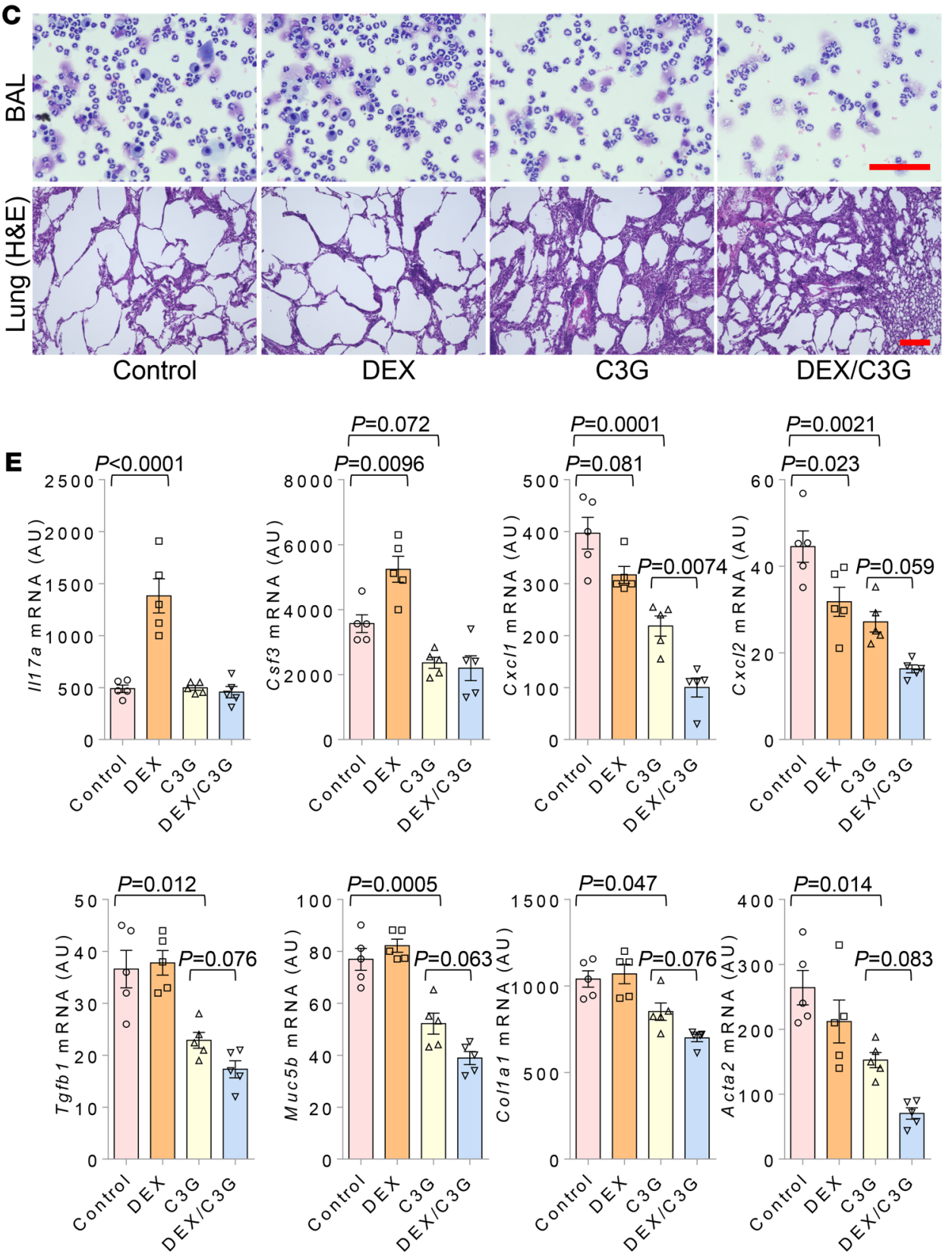
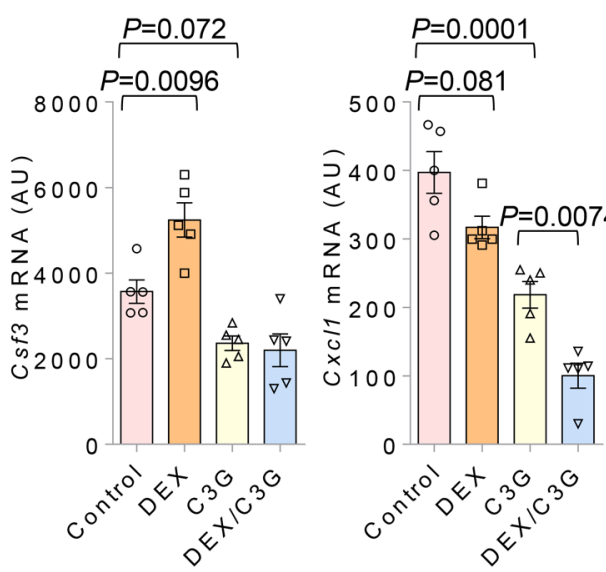

$P=0.0021$

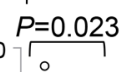

$P=0.023$
$0\urcorner 0$

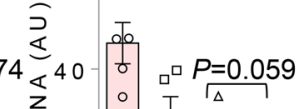

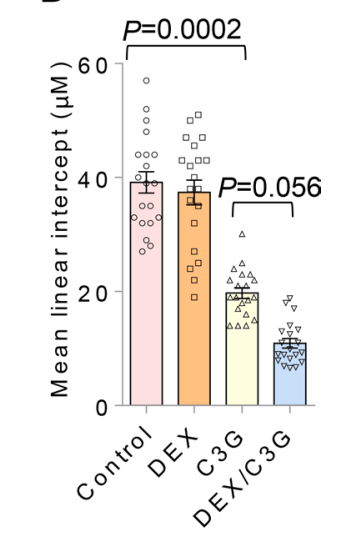

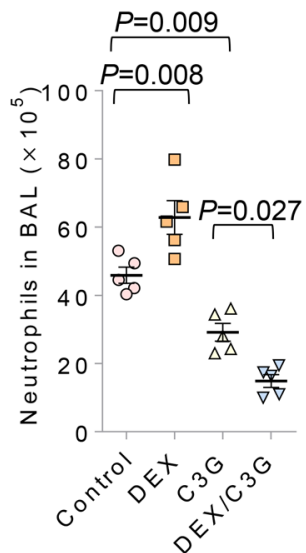

D 
Figure 5. C3G reduces emphysema and restores DEX sensitivity in elastase-induced neutrophilic COPD. (A) Eight-week-old WT C57BL/6 female mice ( $n=5$ mice per group) were subjected to the elastase-induced COPD model. PBS (Control), DEX, C3G, or a combination of DEX and C3G were administered to the mice (as described in Methods). One week after the last challenge, total cell and neutrophil counts in the BAL were quantified (B), and representative BAL samples were prepared by cytospin and lung tissues were stained with H\&E (C). All scale bars (red): $100 \mu \mathrm{m}$. (D) Mean linear intercept (MLI) of airway space was measured by Image). (E) The relative abundances of the indicated mRNAs isolated from lung tissue were determined by real-time PCR. (F) The total collagen content in lung tissue was quantified. COPD, chronic obstructive pulmonary disease; AU, fold induction relative to unchallenged control mice. Data represent mean \pm SEM. One-way ANOVA was performed, followed by Tukey's multiple-comparisons test. The multiplicity-adjusted $P$ values were calculated for the indicated comparisons. For MLI $(n=20)$, Kruskal-Wallis test (nonparametric) was performed, followed by Dunn's multiple-comparisons test. The multiplicity-adjusted $P$ values were calculated for the indicated comparisons. The above data are representative of 3 independent experiments.

Interestingly, this IL-17A/DEX synergy was greatly amplified in the presence of TNF, a widely known IL-17A partner for inflammatory gene expression and frequently upregulated in steroid-resistant severe asthma and COPD upon infections and exacerbations (34-38). Therefore, DEX, especially used at low dose, was unable to resolve or even promoted IL-17A-mediated neutrophilic airway diseases. However, targeting the synergy by blockage of IL-17A signaling with small-molecule inhibitor C3G restored DEX sensitivity, suggesting an effective combined treatment approach (DEX plus C3G) for neutrophilic severe asthma and COPD.

Preclinical studies have provided promising results in murine models of neutrophilic airway diseases by using IL-17A- or IL-17RA-neutralizing antibodies, indicating that IL-17A signaling inhibition could be a novel therapeutic approach for treatment of these steroid-resistant diseases (3, 6-10). Neutralizing monoclonal antibodies (mAbs) (against IL-17A and IL-17RA) have been developed as candidate drugs targeting IL-17A-dependent pathogenesis (39-42). However, given the considerable cost of antibody production and limited administration (only through the i.v. route), our small molecule C3G will likely be a superior cost-effective alternative for treating IL-17A-mediated severe asthma and COPD. Like DEX, C3G has the potential to be formulated for different routes of delivery, including inhalation and pill form for oral intake. Furthermore, small-molecule inhibitors have been shown to have better tissue penetration as compared with antibodies. Thus, our study provides a promising therapeutic strategy (the combined used of low-dose DEX and the small molecule C3G) for treating neutrophilic airway diseases and may have great potential from the viewpoint of drug development.

DEX induced CSF3 expression in ASMCs but the induction level was very limited. However, when partnering with IL-17A, DEX was able to induce CSF3 expression to a much higher magnitude in a synergistic manner in ASMCs, indicating there is a cooperative mechanism between DEX and IL-17A. Human CSF3 promoter-driven luciferase assays suggest that DEX and IL-17A only additively induce CSF3 promoter activity and mapping of the promoter region indicates the respective role of the transcription factors NR3C1 and NF-אB in GC and IL-17A signaling. IL-17A signaling is well known to mediate mRNA stability of inflammatory genes (e.g., CXCL1). IL-17A also stabilized DEX-induced CSF3 mRNA in our study, suggesting that a posttranscriptional mechanism plays a critical role in this IL-17A/DEX synergy. TNF is often upregulated and contributes to steroid-resistant neutrophilic inflammation including severe asthma and COPD (34-37). TNF has been widely reported as an IL-17A partner for synergistic induction of multiple inflammatory genes (e.g., CSF2, CXCL1, CXCL2, CSF3, IL-6, and IL-8) in different cell types (16). Interestingly, we also observed that there existed another layer of synergy between IL-17A/DEX synergy and TNF regarding CSF3 induction in ASMCs. TNF does not stabilize CSF3 mRNA but it synergizes with DEX for CSF3 expression at the transcriptional level. It will be of great interest to further decipher the molecular details of these synergies, which may offer important guidance for developing novel targeting strategies for treating steroid-resistant neutrophilic airway diseases. This study suggests that ASMCs are important CSF3 producers upon DEX and IL-17A stimulation and that IL-17A signaling in ASMCs plays a critical role in the type-17 HDM-CFA steroid-resistant asthma model. In addition, we found that the IL-17A/DEX synergistic effect on CSF3 can also be detected in fibroblasts, suggesting the other cell compartments might also contribute to IL-17A/CSF3-driven steroid resistance in neutrophilic airway diseases.

It should be noted that DEX is known to promote IL-17A production via enhancing Th17 function $(13,25)$ and can hence foster an IL-17A-high environment for IL-17A/DEX synergistic effects on CSF3 expression to take place. Consequently, airway neutrophilia was unaffected or even promoted by DEX treatment due to an antagonizing effect of CSF3 induction on $C X C 1$ and $C X C L 2$ inhibition. The IL-17A blocker C3G not only inhibits IL-17A-induced CXC chemokines and CSF3, it breaks the 
A

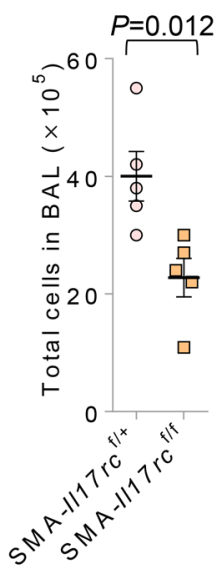

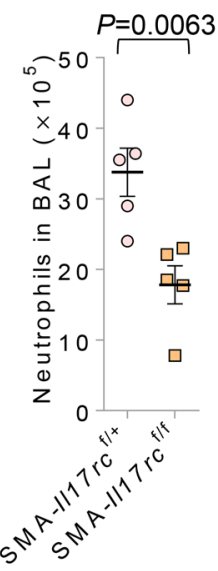

B

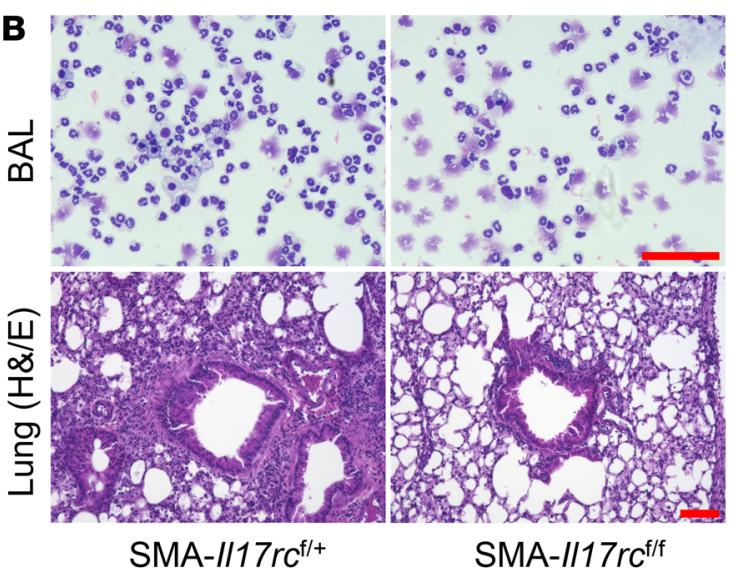

C

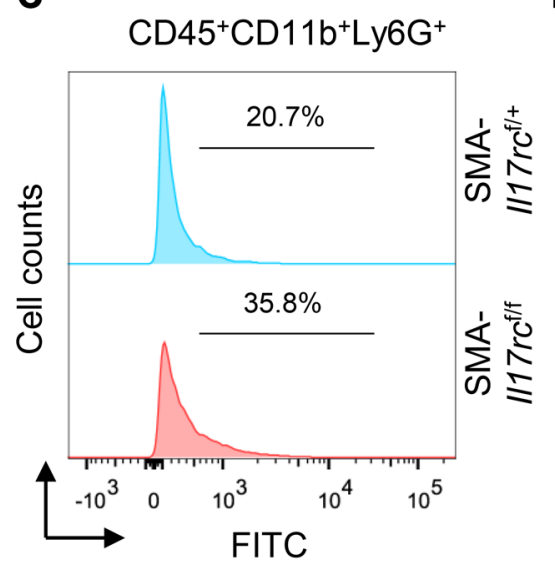

E

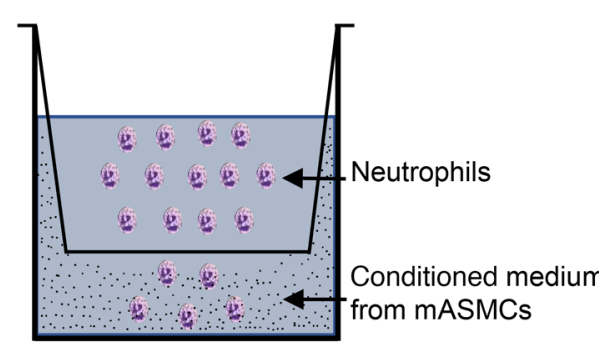

D Early apoptotic cells Late apoptotic cells
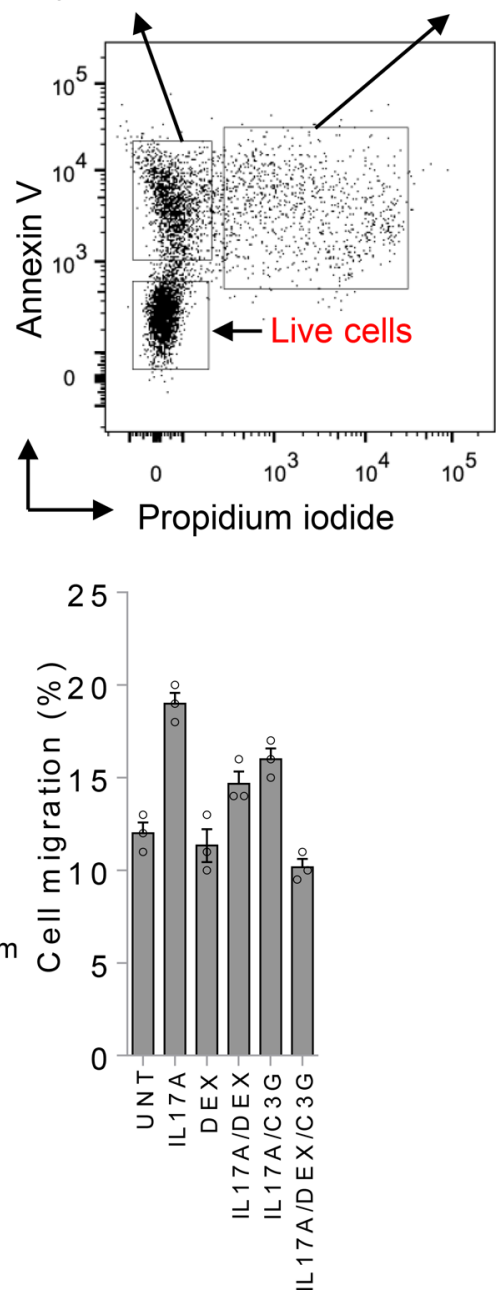

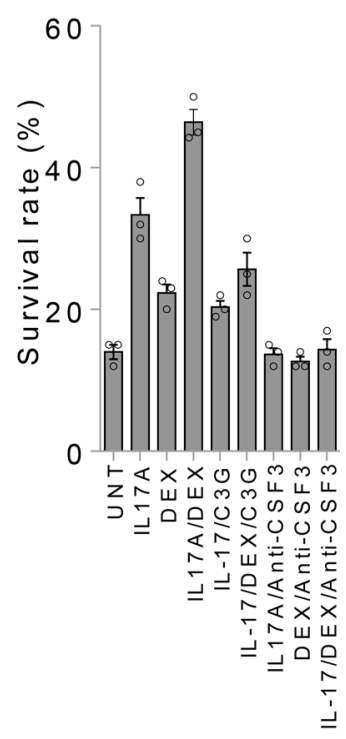

Figure 6. CSF3 induced by IL-17A/DEX in ASMCs is the key neutrophil survival cytokine. (A) Ten-week-old female SMA-specific (Acta2-specific) I/17rc-deficient mice (SMA-I/17r fl/ff) and control mice (SMA-I/17rc $\mathrm{fl}^{\mathrm{fl}+}$ ) were subjected to the HDM-CFA type-17 acute severe asthma mode $(n=5 \mathrm{mice}$ per group). Twenty-four hours after challenging, total and neutrophil counts in the BAL were quantified. Data represent mean \pm SEM. Statistical analysis was performed using 2-tailed Student's $t$ test. (B) Representative BAL cells were prepared by cytospin and lung tissues were stained with H\&E. All scale bars (red): $100 \mu \mathrm{m}$. (C) Lung cells were stained with anti-CD45, anti-CD11b, and anti-Ly6C antibodies, followed by TUNEL assay using biotinylated dNTP and streptavidin-FITC. The apoptotic neutrophils were gated as CD45+CD11 b ${\text { Ly } 6 C^{+} \text {FITC }}^{+}$cells. Representative dot blots from 2 independent experiments are shown. (D) BM-derived neutrophils were cultured in the presence of the conditioned media collected from mouse ASMCs treated for 24 hours as indicated: IL-17A $(100 \mathrm{ng} / \mathrm{mL})$, DEX $(1 \mu \mathrm{M})$, C3C $(100 \mu \mathrm{M})$, anti-CSF3 antibody $(0.5 \mu \mathrm{g} / \mathrm{mL})$. Flow cytometry gating live neutrophils as annexin $\mathrm{V}$ negative and propidium iodide negative. (E) BM-derived neutrophils were loaded into the upper chamber in Transwells, 
and the conditioned media collected from mouse ASMCs treated for 24 hours as indicated were added to the bottom chamber. After 6 hours, the number of migrating neutrophils was counted. For $\mathbf{D}$ and $\mathbf{E}$, data represent mean \pm SD ( $n=3$ technical replicates). The above data are representative of 3 independent experiments.

synergy between DEX and IL-17A so that the CSF3 production is greatly reduced because DEX itself is not a strong inducer of CSF3. Hence, our study provides a strong mechanistic foundation for using combined treatment of low-dose DEX and C3G to treat IL-17A-mediated neutrophilic airway diseases.

As a major effector cell type in the IL-17A/CSF3-mediated chronic asthma model and COPD, neutrophils have been implicated in different pathological features of irreversible airway structural change (airway remodeling). Neutrophils secrete a variety of mediators (e.g., matrix metalloproteinase-9 [MMP9], elastase, YKL-40, growth factors [VEGF, PDGF, and bFGF]) that cause airway remodeling through multiple mechanisms including epithelial damage, fibrosis, ASMC proliferation and migration, and goblet cell metaplasia (43-45). In HDM-CFA chronic asthma and elastase-induced COPD, neutrophil number was associated with many remodeling features in the lung, suggesting that neutrophil inflammation indeed plays a causal role in airway remodeling. In the HDM-CFA type-17 chronic asthma model, C3G can reduce neutrophil-migrating CXCL1 and CXCL2 and disrupt the synergy between IL-17A and DEX on CSF3 expression. Therefore, one importance mechanism by which C3G ameliorates airway remodeling and mitigates DEX resistance is through dampening neutrophilia by reducing the production of neutrophil-promoting cytokines. Targeting IL-17A/CSF3 with DEX/C3G combined treatment is also an effective strategy for alleviating airway remodeling symptoms in chronic asthma and COPD by controlling neutrophilia in the airway.

Our work provides important guidance for designing clinical studies to investigate the pathological role of the IL-17A/CSF3 axis in neutrophilic airway diseases. Single nucleotide polymorphisms (SNPs) of $C S F 3$ have been associated with lung function in smoking-induced COPD in humans (46). Although there were no clinical studies reporting association of CSF3 expression with clinical parameters of asthma, haplotypes (17q21.1) containing the PSMD3-CSF3-MED24 gene region have been reported to be highly associated with adult asthma risk (47). Given the heterogeneous nature of asthma endotypes (e.g., type-2 vs. type-17), it is important to know that future clinical studies need to be appropriately designed by selecting proper patient populations (endotypes) to reveal pathological roles of CSF3 in severe asthma. Our preclinical studies suggest that patients on steroid treatment who have high IL-17A and CSF3 levels may be prone to developing neutrophilic inflammation. Disrupting IL-17A/DEX synergistic effects on CSF3 by anti-IL-17A/C3G or anti-CSF3 treatment is expected be beneficial and render DEX sensitivity for neutrophilic inflammation and associated remodeling features in this subset of asthmatics.

We propose a mechanistic model for improving DEX sensitivity with IL-17A-signaling blockers (antiIL-17A antibody or C3G) in IL-17A-driven neutrophilic inflammation (Figure 7). In IL-17A-mediated neutrophilic severe asthma and COPD, IL-17A is upregulated and induces neutrophil migrating chemokines (CXCL1 and CXCL2) and survival cytokine (CSF3) to elicit neutrophilic inflammation in the airway. DEX treatment can partially reduce expression of $C X C L 1$ and $C X C L 2$ but greatly induce $C S F 3$ expression in synergy with IL-17A and/or other cytokines (e.g., TNF). The dramatically increased neutrophil-survival potential counteracts the reduced neutrophil-migrating potential caused by DEX treatment, which is often not beneficial in neutrophilic inflammation and sometimes may worsen the disease pathology. In the presence of IL-17A-signaling blockers (anti-IL-17A antibody or C3G), IL-17A-induced expression of neutrophil-migrating $C X C L 1$ and $C X C L 2$ was further reduced and the synergy between IL-17A and DEX in affecting CSF3 expression was disrupted so that the DEX-mediated neutrophil-promoting effect was minimized.

\section{Methods}

Mice. B6.OT-II T cell receptor (TCR) transgenic mice (stock no. 004194) and WT C57BL/6 mice (stock no. 000664) were purchased from the Jackson Laboratories. $I l 17 r c^{\mathrm{f} / \mathrm{fl}}$ mice were generated by Cyagen Biosciences using gene-targeting technology. $\alpha$-SMA promoter ( $\alpha$-SMA-rTTA) and (tetO)7-cre mice were obtained from D. Sheppard (UCSF, San Francisco, California, USA). Both strains were described in our previous studies $(28,48)$.

Reagents and cell culture. Mouse TGF- $\beta 1$ (catalog 7666-MB), mouse IL-6 (catalog 406-ML), human IL-17A (catalog 7955-IL-025), mouse IL-17A (catalog 7956-ML-025), mouse TNF- $\alpha$ (catalog 10-MT), and anti-CSF3 neutralizing antibody (catalog MAB414) were purchased from R\&D Systems. Anti-IL-17A neutralizing antibody (clone 17F3), anti-Ly6G antibody (clone 1A8), and isotype control IgG2a (clone 2A3) 


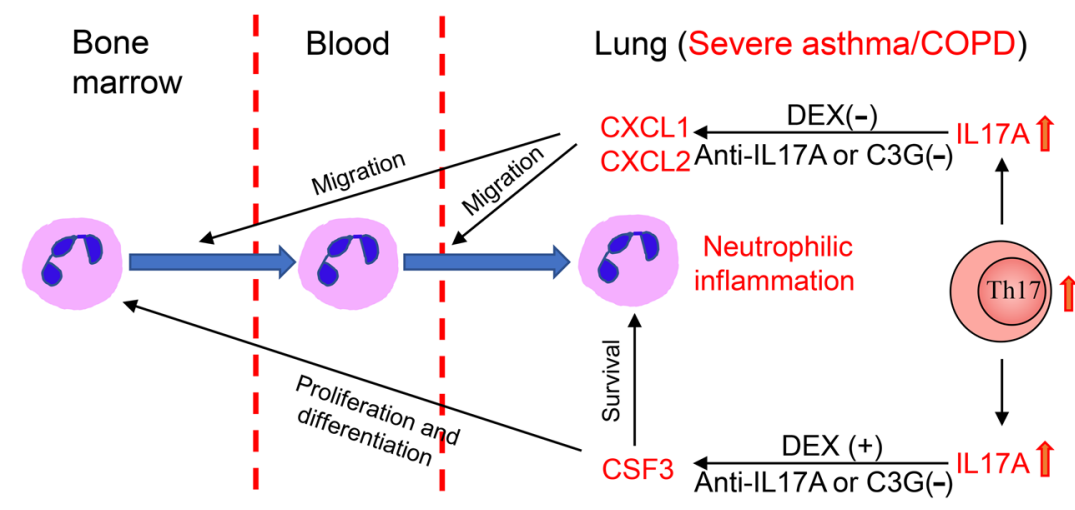

\begin{tabular}{|c|c|c|c|c|}
\hline $\begin{array}{c}\text { Severe } \\
\text { asthma/COPD }\end{array}$ & Treatment & $\begin{array}{l}\text { Neu Migration } \\
\text { (CXCL1/CXCL2) }\end{array}$ & $\begin{array}{l}\text { Neu Survival } \\
\text { (CSF3) }\end{array}$ & $\begin{array}{l}\text { Neutrophilic } \\
\text { Inflammation }\end{array}$ \\
\hline Th17/IL17A & None & ++++ & ++++ & +++++++++ \\
\hline Th17/IL17A & DEX & +++ & ++++++ & $\begin{array}{c}\text { ++++++++++ } \\
\text { (Steroid } \\
\text { resistance) }\end{array}$ \\
\hline Th17/IL17A & Anti-IL17A or C3G & +++ & ++ & +++++ \\
\hline Th17/IL17A & $\begin{array}{c}\text { DEX plus } \\
\text { Anti-IL17A or C3G }\end{array}$ & + & ++ & $\begin{array}{c}+++ \\
\text { (DEX sensitivity } \\
\text { acquired) }\end{array}$ \\
\hline
\end{tabular}

Figure 7. Mechanism for improving DEX sensitivity by IL-17A blockers in IL-17A-mediated neutrophilic airway inflammation. In IL-17A-mediated neutrophilic airway diseases, IL-17A is upregulated and induces neutrophil-migrating chemokines (CXCL1 and CXCL2) and survival cytokine (CSF3) to elicit neutrophilic inflammation in the lung. DEX treatment can partially reduce expression of $C X C L 1$ and $C X C L 2$ but greatly induce CSF3 expression in synergy with IL-17A and/or other cytokines (e.g., TNF). The dramatically increased neutrophil-survival potential counteracts the reduced neutrophil-migrating potential caused by DEX treatment, which is often not beneficial in neutrophilic inflammation and sometimes may worsen the disease pathology. In the presence of IL-17A blockers (anti-IL-17A Ab or C3G), IL-17Ainduced neutrophil-migrating CXCL1 and CXCL2 were further reduced and the synergistic effect between IL-17A and DEX on CSF3 was disrupted so that the DEX-mediated neutrophil-promoting effect was minimized. Neu, neutrophil; red arrow, increase; +, promoting; -, inhibiting.

were obtained from BioXCell. Anti-IL-4 mAb (clone 11B11) and anti-IFN- $\gamma$ mAb (clone XMG1.2) were purchased from BD Biosciences. DEX (catalog sc-29059A) was from Santa Cruz Biotechnology. C3G (cata$\log 2519$ ) was from AvaChem Scientific. CFA (catalog F5881) was from Sigma-Aldrich. A549 cells (ATCC, CCL-185) were maintained in DMEM supplemented with $10 \% \mathrm{FBS}, 100 \mathrm{U} / \mathrm{mL}$ penicillin, and $100 \mu \mathrm{g} /$ $\mathrm{mL}$ streptomycin. Human primary ASMCs were purchased from ATCC (PCS-301-010). Mouse ASMCs were isolated and cultured as described previously (28). Both human and mouse ASMCs were cultured in DMEM/F-12 supplemented with $10 \% \mathrm{FBS}, 100 \mathrm{U} / \mathrm{mL}$ penicillin, and $100 \mu \mathrm{g} / \mathrm{mL}$ streptomycin. Lack of mycoplasma was confirmed for all cell cultures using the Mycoplasma PCR Detection Kit (Sigma-Aldrich, catalog MP0035-1KT). Lung fibroblast isolation was performed as described previously (49). The established fibroblasts were cultured in Eagle's Minimum Essential Medium (ATCC, 30-2003) supplemented with $15 \% \mathrm{FBS}, 100 \mathrm{U} / \mathrm{mL}$ penicillin, and $100 \mu \mathrm{g} / \mathrm{mL}$ streptomycin.

RNA-seq and data analysis. Total RNA extracted by NucleoSpin RNA Plus (Takara Bio, catalog 740984) from human primary ASMCs (untreated and treated with IL-17A [100 $\mathrm{ng} / \mathrm{mL}]$, DEX [1 $\mu \mathrm{g} / \mathrm{mL}]$, or both for 4 hours) was subjected to RNA-seq analysis by Novogene. A total of $2 \mu \mathrm{g}$ RNA per biological replicate (3 per treatment) was used as starting material for the RNA sample preparations. Sequencing libraries were generated using an NEBNext Ultra RNA Library Prep Kit for Illumina, and the clustering of the index-coded samples was performed on a cBot Cluster Generation System using TruSeq PE Cluster Kit v3-cBot-HS (Illumina). The cDNA library preparations were sequenced on an Illumina HiSeq platform to generate 150-bp paired-end reads. HTSeq v0.6.1 was used to count the read numbers mapped to each gene, and FPKM (fragments per kilobase of exon per million mapped fragments of each gene) were calculated. $\log (\mathrm{FPKM})$ values were used to calculate $Z$ scores for generating heatmaps using HemI 1.0. 
Table 1. RT-PCR primers

\begin{tabular}{|c|c|c|}
\hline Gene & Forward primer & Reverse primer \\
\hline \multicolumn{3}{|l|}{ Human } \\
\hline CSF3 & GCTGCTTGAGCCAACTCCATA & GAACGCGGTACGACACCTC \\
\hline CXCL1 & GAAAGCTTGССTСAАTССTG & стTССТССТСССТTСТССTС \\
\hline CXCL2 & GGGCAGAAAGCTTGTCTCAA & GCTTCCTCCTTCCTTCTGGT \\
\hline GAPDH & GTСТССТСТСАСТTСAАСАССG & ACСАСССТСTTССTGTAGССAA \\
\hline \multicolumn{3}{|l|}{ Mouse } \\
\hline$I / 17 a$ & TTTAACTCCCTTGGCGCAAAA & СTTTCССTCСССАTTGACAC \\
\hline II17e & TGGCTCAAGTGGAGCTCTGCAT & CCCGATTCAAGTCCCTGTCCAA \\
\hline 114 & GGTCTCAACCCCCAGCTAGT & GCCGATCATCTCTCTCAAGTGAT \\
\hline 115 & СTCTGTTGACAAGCAATGAGACG & TCTTCAGTATCTCTAGCCCCTC \\
\hline 1113 & ССTGССTСTTССTTСССTT & GGTCTTGTGTCATGTTCСTCA \\
\hline Csf3 & ATGGCTCAАСTTTCTCСССAG & CTGACAGTGACCAGGGGAAC \\
\hline Cxcl1 & TAGGGTGAGGACATCTCTCG & AAATGTCCAAGGGAAGCGT \\
\hline Cxcl2 & CCAACCACCAGGCTACAGG & GCGTCACACTCAAGCTCTG \\
\hline Tgfb1 & сTCCCGTGGCTTCTAGTGC & GCCTTAGTTTGGACAGGATCTC \\
\hline Muc5b & GTGGCCTTGCTCATGGTGT & GGACGAAGGTGACATGCCT \\
\hline Acta2 & GTCCCAGACATCAGGGAGTAA & TCGGATACTTCAGCGTCAGGA \\
\hline Colla1 & GCTCCTCTTAGGGGCCACT & CCACGTCTCACCATTGGGG \\
\hline Gapdh & AGGTCGGTGTGAACGGATTTG & TGTAGACCATGTAGTTGAGGTCA \\
\hline
\end{tabular}

Real-time quantitative PCR. Total RNA was isolated from cells and lung tissues by TRIzol reagent (Invitrogen, catalog 15596026) as per the manufacturer's instructions. First-strand cDNA was synthesized by a SuperScript IV First-Strand Synthesis System (Invitrogen, catalog 18091200). All gene expression results were expressed as AU relative to abundance of GAPDH (human) or Gapdh (mouse) mRNA. The primers used for real-time quantitative PCR (RT-PCR) are as described in Table 1.

CSF3 promoter luciferase assay. LightSwitch human CSF3 promoter luciferase reporter plasmid $(-924 /+89$, catalog S721658) was obtained from Active Motif. WT and different mutant luciferase constructs $(-589 /+89$, $-538 /+89,-198 /+89,-148 /+89$, Del-NR3C1a [-637/-605], Del-NF-kB1 [-660/-636]) were generated by site-directed mutagenesis and transfected into A549 cells for 24 hours, followed by treatment with IL-17A (100 $\mathrm{ng} / \mathrm{mL}), \operatorname{DEX}(1 \mu \mathrm{g} / \mathrm{mL})$, or both for 6 hours. The luciferase activity was analyzed by using a LightSwitch Luciferase Assay kit (Active Motif, catalog 32031) as per the manufacturer's instructions.

ChIP assay. A ChIP Assay Kit (Millipore, catalog 17-295) was used for examining DEX-induced binding of GR to the human CSF3 promoter. Briefly, human ASMCs were grown to $100 \%$ confluence and treated with DEX $(1 \mu \mathrm{M})$ for 30 minutes. Cells were fixed with formaldehyde at a final concentration of $1 \%$ for 10 minutes at $37^{\circ} \mathrm{C}$. Cells were then harvested in SDS lysis buffer and sonicated to shear DNA to a length of 100 to $200 \mathrm{bp}$. Sonicated cell supernatant was then diluted in ChIP dilution buffer and immunoprecipitated with IgG or anti-GR mAb (clone D6H2L, Cell Signaling Technology). DNA was eluted and purified, followed by RT-PCR quantitation with primers (forward, AAGGGTCTTGCCTCATTCGG; reverse, TCGAAAGTTCCAGGCTGTCC) spanning the putative NR3C1a binding site of the CSF3 promoter.

HDM-alum type-2 asthma model. Eight-week-old female WT C57BL/6 mice were sensitized s.c. with HDM (100 $\mu$ g per mouse; Dermatophagoides farina, Greer Laboratories) in Imject Alum (Thermo Fisher Scientific, catalog 77161) on days 0 and 7, and subsequently challenged (i.n.) with HDM (25 $\mu$ g per mouse) from day 14 to day 18 . BAL cell counting and tissue collection were performed on day 21 . DEX $(0.3 \mathrm{mg} / \mathrm{kg}$ per mouse, i.p.) was administered to mice 1 hour before HDM challenge.

HDM-CFA type-17 acute asthma model. This model was described in our previously published work (18). Eight-week-old female WT C57BL/6 mice were sensitized (s.c.) with HDM (100 $\mu$ g per mouse) in CFA (Sigma-Aldrich, F5881) on day 0 and subsequently challenged (i.n.) with HDM (100 $\mu$ g per mouse) on day 14. BAL cell counting and tissue collection were performed 24 hours after the last HDM challenge. DEX ( 0.3 $\mathrm{mg} / \mathrm{kg}$ per mouse, i.p.) and C3G (200 $\mu$ i.p. and $100 \mu \mathrm{g}$ i.n. per mouse) were administered to mice 1 hour before HDM challenge. For cytokine neutralization, IL-17A-neutralizing mAb (100 $\mu$ i.p. per mouse) and CSF3-neutralizing mAb (10 $\mu$ i.p. per mouse) were administered to mice 1 hour before HDM challenge. 
Th17 adoptive transfer model for antigen-induced airway inflammation. OVA-specific Th17 cells were generated as described in our previous study (18). Briefly, naive CD4 ${ }^{+} \mathrm{CD} 44^{-} \mathrm{T}$ cells were isolated from OT-II TCR transgenic mice cultured with irradiated splenic feeder cells in the presence of $5 \mu \mathrm{g} / \mathrm{mL} \mathrm{OVA}_{323-339}$ peptide, $5 \mathrm{ng} / \mathrm{mL}$ TGF- $\beta$, 20 ng/mL IL-6, 10 ng/mL IL-23, $20 \mu \mathrm{g} / \mathrm{mL}$ anti-IL-4 mAb, and $20 \mu \mathrm{g} / \mathrm{mL}$ anti-IFN- $\gamma \mathrm{mAb}$. Th17 cells were then transferred (i.v.) into female WT C57BL/ 6 mice $\left(5 \times 10^{6}\right.$ cells per mouse $)$ and challenged (i.n.) with OVA $_{323-339}(50 \mu \mathrm{g} / \mathrm{mL}) 1$ day before transfer and 3 consecutive days after transfer. BAL cell counting and tissue collection were performed 24 hours after the last challenge. DEX $(0.3 \mathrm{mg} / \mathrm{kg}$ per mouse, i.p.) and C3G (200 $\mu$ g i.p. and $100 \mu \mathrm{g}$ i.n. per mouse) were administered to mice 1 hour before each OVA peptide challenge.

HDM-CFA type-17 chronic asthma model. Eight-week-old female WT C57BL/6 mice were sensitized (s.c.) with HDM (100 $\mu \mathrm{g}$ per mouse) in CFA on day 0 and subsequently challenged (i.n.) with HDM (25 $\mu \mathrm{g}$ per mouse) twice per week for 6 consecutive weeks from day 14. BAL cell counting and lung tissue collection were performed 24 hours after the last HDM challenge. DEX $(0.3 \mathrm{mg} / \mathrm{kg}$ BW per mouse, i.p. $)$ and C3G (200 $\mu$ g i.p. and $100 \mu \mathrm{g}$ i.n. per mouse) were administered to mice 1 hour before each HDM challenge and every other day between each HDM treatment. For IL-17A- and neutrophil-depletion experiments, IL-17A-neutralizing antibody (200 $\mu$ g i.p. per mouse), anti-Ly6G antibody (200 $\mu$ g i.p. per mouse), or an equivalent amount of an isotype control was administered to mice before each allergen challenge during the chronic phase of the HDM-CFA chronic asthma model.

Elastase-induced COPD. Mice were injected (i.n.) with $0.5 \mu \mathrm{g}$ elastase from porcine pancreas (Sigma-A1drich, catalog E1250) in $50 \mu \mathrm{L}$ of saline once per week for 4 consecutive weeks. BAL cell counting and lung tissue collection were performed 24 hours after the last elastase administration. DEX $(0.3 \mathrm{mg} / \mathrm{kg}$ per mouse, i.p.) and C3G (200 $\mu$ i.p. and $100 \mu \mathrm{g}$ i.n. per mouse) were administered to mice 1 hour before each elastase treatment and every other day between each elastase treatment. Airway space enlargement was measured as mean linear intercept (MLI) by ImageJ (NIH) in 20 randomly selected fields.

Differential cell counting and histological staining. BAL cell counts were determined by cytospin slide preparation after a Diff-Quik Giemsa stain. For histological analysis, mouse lung tissue was fixed in $10 \%$ buffered formalin and then stained with H\&E.

Total collagen assay. Total collagen in lung tissue was quantified using a Total Collagen Assay Kit (BioVision, catalog K218-100). Briefly, $10 \mathrm{mg}$ of mouse lung tissue was homogenized with $100 \mu \mathrm{L}$ $\mathrm{ddH}_{2} \mathrm{O}$ and hydrolyzed with $100 \mu \mathrm{L} \mathrm{HCl}(12 \mathrm{M})$ at $120^{\circ} \mathrm{C}$ for 3 hours. After homogenization, samples were clarified with activated charcoal and transferred to 96 -well plates to be evaporated to dryness in an oven. Then, $100 \mu \mathrm{L}$ chloramine $\mathrm{T}$ reagent was added to each sample and incubated at room temperature for 5 minutes, followed by incubation in $100 \mu \mathrm{L}$ 4-(dimethylamino)benzaldehyde (DMAB) reagent for 90 minutes at $60^{\circ} \mathrm{C}$. Finally, the samples were measured at $560 \mathrm{~nm}$ absorbance and total collagen content was calculated using a standard curve.

Collagen staining. A Masson's trichrome staining kit (Abcam, catalog ab15068) was used for collagen staining. The manufacturer's protocol was modified for staining collagen only. Briefly, mouse lung tissues were fixed in $10 \%$ buffered formalin and then embedded in paraffin and sectioned. The section slides were then deparaffinized and hydrated, followed by sequential incubation with phosphomolybdic-phosphotungstic acid and Aniline Blue solution. Then the slides were rinsed with distilled water, dehydrated, cleared, and mounted with cover slides.

PAS staining. Mucins were stained with a PAS staining kit (Sigma-Aldrich, catalog 395B-1KT). Briefly, paraffin sections of mouse lung tissues were deparaffinized, followed by immersion in periodic acid solution for 5 minutes and then in Schiff's reagent for 15 minutes. After washing in running tap water for 5 minutes, the slides were counterstained in hematoxylin solution for 15 seconds.

SMA immunostaining. Briefly, paraffin sections of fixed mouse lung tissues were deparaffinized and blocked with $0.2 \%$ Triton X-100 containing $2 \%$ BSA for 2 hours. Sections were then incubated with mouse anti-SMA-FITC antibody (Sigma-Aldrich, catalog F3377) and rabbit anti-E-cadherin antibody (Cell Signaling Technology, catalog 3195) followed by staining with Alexa Fluor 594-conjugated goat anti-rabbit antibody (Invitrogen, catalog A-1103) and microscopic analysis.

Neutrophil migration and survival assay. Neutrophils were isolated from BM by using a density gradient centrifugation method. Briefly, 8-week-old female WT C57BL/6 mice were used to isolate BM cells from femurs and tibias. BM cells were lysed with ACK lysis buffer (Lonza, catalog 10-548E) to remove red blood cells and resuspended in $3 \mathrm{~mL}$ of ice-cold PBS. The cell suspension was then overlaid over a separation media formed by $3 \mathrm{~mL}$ Histopaque 1077 (Sigma-Aldrich, catalog 10771) and $3 \mathrm{~mL}$ Histopaque 1119 (Sigma-Aldrich, catalog 11191) in 
a $15-\mathrm{mL}$ tube-column. The column was centrifuged at $1000 \mathrm{~g}$ for 30 minutes at $25^{\circ} \mathrm{C}$ and the neutrophil layer (containing $>95 \% \mathrm{CD}_{4} 5^{+} \mathrm{Ly}_{6 \mathrm{G}^{+}}$cells) at the interphase of the 2 Histopaques was collected and washed for migration and survival assay. For migration assay, a total of $1 \times 10^{6}$ neutrophils were added to the upper chambers of Transwells ( $3 \mu \mathrm{m}$ pore) (Corning, catalog 3415$)$ and migration to the lower chamber was stimulated for 8 hours at $37^{\circ} \mathrm{C}$ in the presence of conditioned medium collected from mouse ASMCs subjected to different treatments. The transmigrated cells were collected from the lower chamber and counted. For survival assays, BM neutrophils were cultured with conditioned medium collected from mouse ASMCs for 24 hours in the presence of GR inhibitor A1082D06 (CAS no. 256925-03-8, Cayman Chemical). The cells were stained using a FITC-Annexin V Apoptosis Detection Kit I (BD Biosciences, catalog 556547) with live cells gated as negative for both annexin $\mathrm{V}$ and propidium iodide (PI) by BD LSRFortessa cell analyzer (BD Biosciences).

TUNEL assay of lung neutrophils. PBS-perfused lung tissues were cut into small pieces and subjected to 45-minute digestion at $37^{\circ} \mathrm{C}$ with Liberase (Millipore, catalog 5401119001) plus DNase I (Millipore, catalog 11284932001). Single-cell solutions were obtained using cell strainers $(40 \mu \mathrm{m})$, followed by treatment with RBC lysis buffer (BioLegend, catalog 420301). The cells were blocked with anti-mouse CD16/32 mAb (clone 2.4G, BD Biosciences, catalog 553142) to avoid nonspecific binding to Fc receptors, followed by staining with AF700-anti-CD45 (BioLegend, catalog 103128), PE-CF594-anti-CD11b (BD Biosciences, catalog 562317), and APC-anti-Ly6G (BioLegend, catalog 127614) antibodies for gating neutrophils. Then the cells were pelleted for TUNEL assay using a FlowTACS Apoptosis Detection Kit (R\&D Systems, catalog 4817 60-K). Briefly, the cells were fixed in $1 \mathrm{~mL} \mathrm{3.7 \%} \mathrm{formaldehyde} \mathrm{for} 5$ minutes and incubated in Cytonin solution for 30 minutes. After washing with labeling buffer, the cells were resuspended in labeling reaction mix (composed of $\mathrm{MnCl}_{2}$, biotinylated dNTP, and terminal deoxynucleotidyl transferase enzyme) and incubated for 1 hour at $37^{\circ} \mathrm{C}$. The cells were washed with stop buffer and stained with streptavidin-FITC for 10 minutes. The apopototic neutrophils were gated as $\mathrm{CD} 45^{+} \mathrm{CD} 11 \mathrm{~b}^{+} \mathrm{Ly}_{6 \mathrm{G}}{ }^{+} \mathrm{FITC}{ }^{+}$cells by BD LSRFortessa. Dead cells were excluded using Zombie NIR Fixable Viability dye (BioLegend, catalog 423105).

Statistics. Sample size and distribution were determined based on preliminary data and previous statistical analyses of the experimental model. Unless otherwise specified, we performed 1-way ANOVA (parametric) followed by Tukey's multiple-comparisons test or Kruskal Wallis test (non-parametric) followed by Dunn's multiple-comparisons test. The multiplicity-adjusted $P$ values are reported for the indicated comparisons. Spearman's rank correlation test was performed for correlation analysis. All tests and calculations were performed using GraphPad Prism (version 6).

Study approval. All animal studies were performed in accordance with the guidelines of and approved by the IACUC of the Cleveland Clinic.

Accession codes. RNA-seq data were deposited in the NCBI's Gene Expression Omnibus database (GEO GSE135730.

\section{Author contributions}

$\mathrm{CL}$ and XL conceived and designed all the experiments. SO, CL, JX, XC, and ACL performed the experiments. CL and XL wrote the manuscript. XL supervised all aspects of the study.

\section{Acknowledgments}

This study was supported by the NIH (grants R01NS071996, P01 CA062220-21 project 3, P01 HL103453 project 2, and 1UH54HL119810-01 to XL) and the American Asthma Foundation (18-LIXI-EX1 to XL).

Address correspondence to: Xiaoxia Li, Inflammation and Immunity, Cleveland Clinic, 9500 Euclid Avenue, Cleveland, Ohio 44195, USA. Phone: 216.952.2437; Email: lix@ccf.org.

1. Le Rouzic O, Pichavant M, Frealle E, Guillon A, Si-Tahar M, Gosset P. Th17 cytokines: novel potential therapeutic targets for COPD pathogenesis and exacerbations. Eur Respir J. 2017;50(4):1602434.

2. Roos AB, Stampfli MR. Targeting Interleukin-17 signalling in cigarette smoke-induced lung disease: Mechanistic concepts and therapeutic opportunities. Pharmacol Ther. 2017;178:123-131.

3. Yanagisawa H, et al. Role of IL-17A in murine models of COPD airway disease. Am J Physiol Lung Cell Mol Physiol. 2017;312(1):L122-L130.

4. Alcorn JF, Crowe CR, Kolls JK. TH17 cells in asthma and COPD. Annu Rev Physiol. 2010;72:495-516.

5. Chesné J, Braza F, Mahay G, Brouard S, Aronica M, Magnan A. IL-17 in severe asthma. Where do we stand? Am J Respir Crit Care Med. 2014;190(10):1094-1101. 
6. Busse WW, et al. Randomized, double-blind, placebo-controlled study of brodalumab, a human anti-IL-17 receptor monoclonal antibody, in moderate to severe asthma. Am J Respir Crit Care Med. 2013;188(11):1294-1302.

7. Choy DF, et al. TH2 and TH17 inflammatory pathways are reciprocally regulated in asthma. Sci Transl Med. 2015;7(301):301ra129.

8. Shen N, Wang J, Zhao M, Pei F, He B. Anti-interleukin-17 antibodies attenuate airway inflammation in tobacco-smoke-exposed mice. Inhal Toxicol. 2011;23(4):212-218.

9. Schnyder-Candrian S, et al. Interleukin-17 is a negative regulator of established allergic asthma. J Exp Med. 2006;203(12):2715-2725

10. Chesné J, et al. Prime role of IL-17A in neutrophilia and airway smooth muscle contraction in a house dust mite-induced allergic asthma model. J Allergy Clin Immunol. 2015;135(6):1643-1643.e3.

11. McMaster A, Ray DW. Modelling the glucocorticoid receptor and producing therapeutic agents with anti-inflammatory effects but reduced side-effects. Exp Physiol. 2007;92(2):299-309.

12. Oakley RH, Cidlowski JA. The biology of the glucocorticoid receptor: new signaling mechanisms in health and disease. $J$ Allergy Clin Immunol. 2013;132(5):1033-1044.

13. McKinley L, et al. TH17 cells mediate steroid-resistant airway inflammation and airway hyperresponsiveness in mice. $J$ Immunol. 2008;181(6):4089-4097.

14. Brandt EB, Khurana Hershey GK. A combination of dexamethasone and anti-IL-17A treatment can alleviate diesel exhaust particle-induced steroid insensitive asthma. J Allergy Clin Immunol. 2016;138(3):924-928.e2.

15. Gross NJ, Barnes PJ. New therapies for asthma and chronic obstructive pulmonary disease. Am J Respir Crit Care Med. 2017;195(2):159-166.

16. McGeachy MJ, Cua DJ, Gaffen SL. The IL-17 family of cytokines in health and disease. Immunity. 2019;50(4):892-906.

17. Khan A, et al. JASPAR 2018: update of the open-access database of transcription factor binding profiles and its web framework. Nucleic Acids Res. 2018;46(D1):D1284.

18. Liu C, et al. The flavonoid cyanidin blocks binding of the cytokine interleukin-17A to the IL-17RA subunit to alleviate inflammation in vivo. Sci Signal. 2017;10(467):eaaf8823.

19. Czank C, et al. Human metabolism and elimination of the anthocyanin, cyanidin-3-glucoside: a (13)C-tracer study. Am J Clin Nutr. 2013;97(5):995-1003.

20. de Ferrars RM, et al. The pharmacokinetics of anthocyanins and their metabolites in humans. Br J Pharmacol. 2014;171(13):3268-3282.

21. Chakir J, et al. Airway remodeling-associated mediators in moderate to severe asthma: effect of steroids on TGF-beta, IL-11, IL-17, and type I and type III collagen expression. J Allergy Clin Immunol. 2003;111(6):1293-1298.

22. Mi S, et al. Blocking IL-17A promotes the resolution of pulmonary inflammation and fibrosis via TGF-beta1-dependent and -independent mechanisms. J Immunol. 2011;187(6):3003-3014.

23. Chang Y, et al. Th17-associated cytokines promote human airway smooth muscle cell proliferation. FASEBJ. 2012;26(12):5152-5160.

24. Fogli LK, et al. T cell-derived IL-17 mediates epithelial changes in the airway and drives pulmonary neutrophilia. J Immunol. 2013;191(6):3100-3111.

25. Zhao J, Lloyd CM, Noble A. Th17 responses in chronic allergic airway inflammation abrogate regulatory T-cell-mediated tolerance and contribute to airway remodeling. Mucosal Immunol. 2013;6(2):335-346.

26. Zou Y, et al. Serum IL-1 $\beta$ and IL-17 levels in patients with COPD: associations with clinical parameters. Int J Chron Obstruct Pulmon Dis. 2017;12:1247-1254.

27. Voss M, et al. Il-17A contributes to maintenance of pulmonary homeostasis in a murine model of cigarette smoke-induced emphysema. Am J Physiol Lung Cell Mol Physiol. 2015;309(2):L188-L195.

28. Bulek K, et al. IL-17A recruits Rab35 to IL-17R to mediate PKC $\alpha$-dependent stress fiber formation and airway smooth muscle contractility. J Immunol. 2019;202(5):1540-1548.

29. Liles WC, Dale DC, Klebanoff SJ. Glucocorticoids inhibit apoptosis of human neutrophils. Blood. 1995;86(8):3181-3188.

30. Miner JN, et al. A nonsteroidal glucocorticoid receptor antagonist. Mol Endocrinol. 2003;17(1):117-127.

31. Ellis EF. Adverse effects of corticosteroid therapy. J Allergy Clin Immunol. 1987;80(4):515-517.

32. Fitzsimons R, Grammer LC, Halwig JM, Aksamit T, Patterson R. Prevalence of adverse effects in corticosteroid dependent asthmatics. N Engl Reg Allergy Proc. 1988;9(2):157-162.

33. Sullivan PW, Ghushchyan VH, Globe G, Schatz M. Oral corticosteroid exposure and adverse effects in asthmatic patients. $J$ Allergy Clin Immunol. 2018;141(1):110-116.e7.

34. de Godoy I, Donahoe M, Calhoun WJ, Mancino J, Rogers RM. Elevated TNF-alpha production by peripheral blood monocytes of weight-losing COPD patients. Am J Respir Crit Care Med. 1996;153(2):633-637.

35. Howarth $\mathrm{PH}$, et al. Tumour necrosis factor (TNFalpha) as a novel therapeutic target in symptomatic corticosteroid dependent asthma. Thorax. 2005;60(12):1012-1018.

36. Berry MA, et al. Evidence of a role of tumor necrosis factor alpha in refractory asthma. N Engl J Med. 2006;354(7):697-708.

37. Antoniu SA. Challenges in targeting TNF alpha in asthma and COPD. Curr Opin Investig Drugs. 2009;10(5):404-406.

38. Pomerenke A, Lea SR, Herrick S, Lindsay MA, Singh D. Characterization of TLR-induced inflammatory responses in COPD and control lung tissue explants. Int J Chron Obstruct Pulmon Dis. 2016;11:2409-2417.

39. Amin M, Darji K, No DJ, Bhutani T, Wu JJ. Review of IL-17 inhibitors for psoriasis. J Dermatolog Treat. 2018;29(4):347-352.

40. Patel NU, Vera NC, Shealy ER, Wetzel M, Feldman SR. A review of the use of secukinumab for psoriatic arthritis. Rheumatol Ther. 2017;4(2):233-246.

41. Puig L. Brodalumab: the first anti-IL-17 receptor agent for psoriasis. Drugs Today. 2017;53(5):283-297.

42. Roostaeyan O, Kivelevitch D, Menter A. A review article on brodalumab in the treatment of moderate-to-severe plaque psoriasis. Immunotherapy. 2017;9(12):963-978.

43. Gao H, Ying S, Dai Y. Pathological roles of neutrophil-mediated inflammation in asthma and its potential for therapy as a target. J Immunol Res. 2017;2017:3743048.

44. Saglani S, Lloyd CM. Novel concepts in airway inflammation and remodelling in asthma. Eur Respir J. 2015;46(6):1796-1804.

45. Fehrenbach H, Wagner C, Wegmann M. Airway remodeling in asthma: what really matters. Cell Tissue Res. 2017;367(3):551-569. 
46. He JQ, Shumansky K, Connett JE, Anthonisen NR, Paré PD, Sandford AJ. Association of genetic variations in the CSF2 and CSF3 genes with lung function in smoking-induced COPD. Eur Respir J. 2008;32(1):25-34

47. Žavbi M, Korošec P, Fležar M, Škrgat Kristan S, Marc Malovrh M, Rijavec M. Polymorphisms and haplotypes of the chromosome locus 17q12-17q21.1 contribute to adult asthma susceptibility in Slovenian patients. Hum Immunol. 2016;77(6):527-534.

48. $\mathrm{Wu} \mathrm{L}$, et al. A novel IL-17 signaling pathway controlling keratinocyte proliferation and tumorigenesis via the TRAF4-ERK5 axis. J Exp Med. 2015;212(10):1571-1587.

49. Seluanov A, Vaidya A, Gorbunova V. Establishing primary adult fibroblast cultures from rodents. J Vis Exp. 2010;(44):2033. 